\title{
Nonlinear and Linear Conservative Finite Difference Schemes for Regularized Long Wave Equation
}

\author{
Satoshi KoIDE* and Daisuke Furihata ${ }^{\dagger}$ \\ * Department of Pure and Applied Mathematics \\ Graduate School of Information Science and Technology, Osaka University \\ Machikaneyama 1-1, Toyonaka, Osaka 560-0021, Japan \\ E-mail: satoshi-k@cas.cmc.osaka-u.ac.jp \\ †Cybermedia Center, Osaka University \\ Machikaneyama 1-32, Toyonaka, Osaka 560-0043, Japan \\ E-mail: furihata@cmc.osaka-u.ac.jp
}

Received April 5, 2006

Revised September 1, 2008

\begin{abstract}
We propose four conservative schemes for the regularized long-wave (RLW) equation. The RLW equation has three invariants: mass, momentum, and energy. Our schemes are designed by using the discrete variational derivative method to inherit appropriate conservation properties from the equation. Two of our schemes conserve mass and momentum, while the other two schemes conserve mass and energy. With one of our schemes, we prove the numerical solution stability, the existence of the solutions, and the convergence of the solutions. Through some numerical computation examples, we demonstrate the efficiency and robustness of our schemes.
\end{abstract}

Key words: finite difference method, discrete conservation laws, nonlinear and linear schemes, RLW equation

\section{Introduction}

In this study we propose four conservative schemes for the regularized long wave (RLW) equation for $u=u(x, t)$,

$$
\left(1-\frac{\partial^{2}}{\partial x^{2}}\right) \frac{\partial u}{\partial t}=-\frac{\partial}{\partial x}\left(\frac{\delta G}{\delta u}\right), \quad x \in \mathbf{R}, t>0,
$$

where

$$
\frac{\delta G}{\delta u} \stackrel{\text { def }}{=} u+\frac{1}{2} u^{2} .
$$

For this equation we adopt periodic boundary conditions with a virtual length $L>0$,

$$
\frac{\partial^{l}}{\partial x^{l}} \frac{\partial^{m}}{\partial t^{m}} u(x+k L, t)=\frac{\partial^{l}}{\partial x^{l}} \frac{\partial^{m}}{\partial t^{m}} u(x, t), \quad l=0,1, m=0,1,
$$

for any $k \in \mathbf{Z}, x \in \mathbf{R}$, and $t>0$.

This equation, also known as the Benjamin-Bona-Mahony (BBM) equation, has been proposed as a model equation for the undular bore problem by Peregrine [17]. Benjamin et al. [1] have investigated this equation as a regularized version of the Korteweg-de Vries (KdV) equation. This equation has solitary wave 
solutions which are similar to those of the KdV equations. Although the KdV equation has an infinite number of conserved quantities, Olver [16] has proved that the RLW equation admits only three independent conserved quantities:

$$
\begin{aligned}
& M[u(\cdot, t)] \stackrel{\text { def }}{=} \int_{0}^{L} u(x, t) \mathrm{d} x, \\
& I[u(\cdot, t)] \stackrel{\text { def }}{=} \frac{1}{2} \int_{0}^{L}\left\{u^{2}+\left(\frac{\partial u}{\partial x}\right)^{2}\right\} \mathrm{d} x, \\
& J[u(\cdot, t)] \stackrel{\text { def }}{=} \frac{1}{2} \int_{0}^{L}\left(u^{2}+\frac{1}{3} u^{3}\right) \mathrm{d} x .
\end{aligned}
$$

Here, $M, I$, and $J$ are called 'mass,' 'momentum,' and 'energy.' Since the number of conserved quantities is limited, we are not able to use the inverse-scattering technique, which is a powerful mathematical tool to obtain the theoretical solutions of integrable equations such as the KdV equation.

A large number of studies have been carried out in order to obtain the numerical solutions of the RLW equation $[1,2,3,4,5,6,17]$. For example, Peregrine [17] has proposed a simple finite difference scheme, which is first-order accurate in time. Further, Eilbeck and McGuire [5, 6] have proposed a linear scheme and a nonlinear scheme, which are second-order accurate schemes. Dă̆ and Özer [2] have used a finite element method based on cubic and quadratic B-splines. Dağ et al. [3] have used a cubic B-spline collocation method to find the numerical solutions of the RLW equation. Durán and López-Marcos [4] have investigated some advantages of conservative numerical methods. Although Durán and López-Marcos scheme conserves momentum, it is different from our proposed scheme described in the next section. For a brief summary of the computational studies on the RLW equation, refer [3].

We propose four finite difference schemes for the RLW equation. These schemes are constructed using the discrete variational derivative method (DVDM), which is a methodology used to design conservative numerical schemes. Furihata and Mori [10] have used the DVDM to design a stable finite difference scheme for the Cahn-Hilliard equation. Furihata [7, 8] has carried out some general studies on the DVDM. Further, Matsuo and Furihata [14] have extended the general studies to include complex-valued PDEs such as the nonlinear Schrödinger equation. Hanada et al. [11] have used the DVDM to design a numerical scheme for the Eguchi-OkiMatsumura equation, and Ide et al. [12] have used the DVDM for the numerical solutions of the Fujita problem. For a standard study on the DVDM, refer [7]. For more details on the proposed linear schemes, refer [9].

This paper is structured as follows. In Section 2, we propose the four finite difference schemes and describe their conservations properties. In Section 3, we show three important features of our proposed momentum-conserving scheme. The three features are solution stability, existence, and convergence. In Section 4, we present some numerical computation examples using our proposed schemes. 


\section{Four proposed finite difference schemes}

In this section, we propose the four finite difference schemes that conserve either discrete momentum or discrete energy. Two of them are nonlinear schemes, while the other two are linear schemes. As described in the introduction, they have been designed using the DVDM.

We define $U_{k}^{(n)}(k \in \mathbf{Z}, n=0,1,2, \ldots)$ as the approximation to $u(x, t)$ at location $x=k \Delta x$ and time $t=n \Delta t$, where $\Delta x=L / N(N \in \mathbf{Z})$ is the space mesh size and $\Delta t$ is the time mesh size. For the computation, we adopt the following discrete periodic boundary conditions:

$$
U_{k}^{(n)}=U_{k \bmod N}^{(n)} \quad \text { for } \forall k \in \mathbf{Z} .
$$

We assume that the numerical solutions $U_{k}^{(n)}$ always satisfy the abovementioned condition. For simplicity, we denote a variable set under the abovementioned boundary condition by

$$
\mathbf{R}^{(N)} \stackrel{\text { def }}{=}\left\{\boldsymbol{f}=\left\{f_{k}\right\}_{k \in \mathbf{Z}} \mid f_{k} \in \mathbf{R}, f_{k}=f_{k \bmod N} \text { for } \forall k \in \mathbf{Z}\right\} .
$$

\subsection{Concrete form of proposed schemes}

The concrete form of the first proposed finite difference scheme, which conserves momentum and mass, is given by

$$
\left(1-\delta_{k}^{\langle 2\rangle}\right) \delta_{n}^{+} U_{k}^{(n)}=-\delta_{k}^{\langle 1\rangle}\left(\frac{\delta G_{\mathrm{d}}}{\delta\left(\boldsymbol{V}_{+}^{(n)}, \boldsymbol{V}_{-}^{(n)}\right)}\right)_{k}, \quad k \in \mathbf{Z}, n=0,1, \ldots,
$$

where $\frac{\delta G_{\mathrm{d}}}{\delta(\boldsymbol{f}, \boldsymbol{g})}$ is an $\mathbf{R}^{(N)} \times \mathbf{R}^{(N)} \rightarrow \mathbf{R}^{(N)}$ function given as follows:

$$
\begin{aligned}
& \left(\frac{\delta G_{\mathrm{d}}}{\delta(\boldsymbol{f}, \boldsymbol{g})}\right)_{k} \stackrel{\text { def }}{=}\left(\frac{f_{k}+g_{k}}{2}\right)+\frac{1}{2}\left(\frac{\left(f_{k}\right)^{2}+f_{k} g_{k}+\left(g_{k}\right)^{2}}{3}\right), \quad k \in \mathbf{Z}, \\
& \left(\boldsymbol{V}_{+}^{(n)}\right)_{k} \stackrel{\text { def }}{=} \frac{1}{2}\left(U_{k+1}^{(n+1)}+U_{k+1}^{(n)}\right), \quad\left(\boldsymbol{V}_{-}^{(n)}\right)_{k} \stackrel{\text { def }}{=} \frac{1}{2}\left(U_{k-1}^{(n+1)}+U_{k-1}^{(n)}\right),
\end{aligned}
$$

$k \in \mathbf{Z}, n=0,1, \ldots$

Here, $\delta_{k}^{\langle 2\rangle}$ is a second-order difference operator defined by $\delta_{k}^{\langle 2\rangle} f_{k} \stackrel{\text { def }}{=}\left(f_{k-1}-2 f_{k}+\right.$ $\left.f_{k+1}\right) /(\Delta x)^{2}, \delta_{k}^{\langle 1\rangle}$ is a first-order difference operator defined by $\delta_{k}^{\langle 1\rangle} f_{k} \stackrel{\text { def }}{=}\left(f_{k+1}-\right.$ $\left.f_{k-1}\right) /(2 \Delta x)$ and $\delta_{n}^{+}$is a forward difference operator defined by $\delta_{n}^{+} g^{(n)}=\left(g^{(n+1)}-\right.$ $\left.g^{(n)}\right) / \Delta t$. We note that $\boldsymbol{V}_{+}^{(n)}, \boldsymbol{V}_{-}^{(n)} \in \mathbf{R}^{(N)}$ and $\frac{\delta G_{\mathrm{d}}}{\delta\left(\boldsymbol{V}_{+}^{(n)}, \boldsymbol{V}_{-}^{(n)}\right)} \in \mathbf{R}^{(N)}$.

Using the scheme (9), we have to solve nonlinear equations to obtain a new time-step solution, $\boldsymbol{U}^{(n+1)}$. Because of this nonlinearity and the conservation of momentum we call the scheme (9) as the nonlinear momentum-conserving (NM) scheme. 
REMARK 1. $\frac{\delta G_{\mathrm{d}}}{\delta(\boldsymbol{f}, \boldsymbol{g})}$ is a discrete variational derivative of the function

$$
G_{\mathrm{d}}(\boldsymbol{f})_{k} \stackrel{\text { def }}{=} \frac{1}{2}\left(f_{k}\right)^{2}+\frac{1}{6}\left(f_{k}\right)^{3},
$$

and they satisfy

$$
\sum_{k=0}^{N-1}\left\{G_{\mathrm{d}}(\boldsymbol{f})_{k}-G_{\mathrm{d}}(\boldsymbol{g})_{k}\right\} \Delta x=\sum_{k=0}^{N-1}\left(\frac{\delta G_{\mathrm{d}}}{\delta(\boldsymbol{f}, \boldsymbol{g})}\right)_{k}\left(f_{k}-g_{k}\right) \Delta x
$$

The second scheme is also a nonlinear scheme. Since it conserves energy and mass, we call it as the nonlinear energy-conserving (NE) scheme. It is given as

$$
\left(1-\delta_{k}^{\langle 1\rangle} \delta_{k}^{\langle 1\rangle}\right) \delta_{n}^{+} U_{k}^{(n)}=-\delta_{k}^{\langle 1\rangle}\left(\frac{\delta G_{\mathrm{d}}}{\delta\left(\boldsymbol{U}^{(n+1)}, \boldsymbol{U}^{(n)}\right)}\right)_{k}, \quad k \in \mathbf{Z}, n=0,1, \ldots
$$

The third scheme conserves momentum and mass, but we do not have to solve nonlinear equations to obtain a new time-step solution. To obtain the solution, we have to solve linear equations; hence, we call it as the linear momentum-conserving (LM) scheme. Its forms given by

$$
\left(1-\delta_{k}^{\langle 2\rangle}\right) \delta_{n}^{\langle 1\rangle} U_{k}^{(n)}=-\delta_{k}^{\langle 1\rangle}\left(\frac{\delta G_{\mathrm{d}}}{\delta\left(\boldsymbol{U}_{+}^{(n)}, \boldsymbol{U}_{-}^{(n)}\right)}\right)_{k}, \quad k \in \mathbf{Z}, n=1,2, \ldots,
$$

where

$$
\left(\boldsymbol{U}_{+}^{(n)}\right)_{k} \stackrel{\text { def }}{=} U_{k+1}^{(n)}, \quad\left(\boldsymbol{U}_{-}^{(n)}\right)_{k} \stackrel{\text { def }}{=} U_{k-1}^{(n)}, \quad k \in \mathbf{Z}, n=0,1, \ldots
$$

Here, $\delta_{n}^{\langle 1\rangle}$ is a first-order difference operator defined by $\delta_{n}^{\langle 1\rangle} g^{(n)} \stackrel{\text { def }}{=}\left(g^{(n+1)}-\right.$ $\left.g^{(n-1)}\right) /(2 \Delta t)$.

The last scheme is a linear scheme that conserves energy and mass; hence, we call it as the linear energy-conserving (LE) scheme. The form of the scheme is given by

$$
\left(1-\delta_{k}^{\langle 1\rangle} \delta_{k}^{\langle 1\rangle}\right) \delta_{n}^{\langle 1\rangle} U_{k}^{(n)}=-\delta_{k}^{\langle 1\rangle}\left(\frac{\delta \tilde{G}_{\mathrm{d}}}{\delta\left(\boldsymbol{U}^{(n+1)}, \boldsymbol{U}^{(n)}, \boldsymbol{U}^{(n-1)}\right)}\right)_{k}, \quad k \in \mathbf{Z}, n=1,2, \ldots,
$$

where $\frac{\delta \tilde{G}_{\mathrm{d}}}{\delta(\boldsymbol{f}, \boldsymbol{g}, \boldsymbol{h})}$ is an $\mathbf{R}^{(N)} \times \mathbf{R}^{(N)} \times \mathbf{R}^{(N)} \rightarrow \mathbf{R}^{(N)}$ function and

$$
\left(\frac{\delta \tilde{G}_{\mathrm{d}}}{\delta(\boldsymbol{f}, \boldsymbol{g}, \boldsymbol{h})}\right)_{k} \stackrel{\text { def }}{=} g_{k}+\frac{1}{2}\left(\frac{\left(f_{k}+g_{k}+h_{k}\right) g_{k}}{3}\right), \quad k \in \mathbf{Z} .
$$


REMARK 2. $\frac{\delta \tilde{G}_{\mathrm{d}}}{\delta(\boldsymbol{f}, \boldsymbol{g}, \boldsymbol{h})}$ is a discrete variational derivative of the function

$$
\tilde{G}_{\mathrm{d}}(\boldsymbol{f}, \boldsymbol{g})_{k} \stackrel{\text { def }}{=} \frac{1}{2} f_{k} g_{k}+\frac{1}{6}\left(\frac{\left(f_{k}\right)^{2} g_{k}+f_{k}\left(g_{k}\right)^{2}}{2}\right),
$$

and they satisfy

$$
\sum_{k=0}^{N-1}\left\{\tilde{G}_{\mathrm{d}}(\boldsymbol{f}, \boldsymbol{g})_{k}-\tilde{G}_{\mathrm{d}}(\boldsymbol{g}, \boldsymbol{h})_{k}\right\} \Delta x=\sum_{k=0}^{N-1}\left(\frac{\delta \tilde{G}_{\mathrm{d}}}{\delta(\boldsymbol{f}, \boldsymbol{g}, \boldsymbol{h})}\right)_{k}\left(\frac{f_{k}-h_{k}}{2}\right) \Delta x .
$$

\subsection{Conservation properties of proposed schemes}

We first study three conservation properties of the solution of the RLW equation, which are mentioned in the introduction, namely,

$$
\begin{aligned}
\frac{d}{d t} M[u(\cdot, t)] & =0, \\
\frac{d}{d t} I[u(\cdot, t)] & =0, \\
\frac{d}{d t} J[u(\cdot, t)] & =0 .
\end{aligned}
$$

Equations (21), (22), and (23) are called the equations of mass conservation, momentum conservation, and energy conservation, respectively. Under the periodic boundary condition, the conservation of mass (21) can be easily proved as follows:

$\frac{d}{d t} M[u(\cdot, t)]=\int_{0}^{L} \frac{\partial u}{\partial t} \mathrm{~d} x=\int_{0}^{L}\left\{\frac{\partial^{2}}{\partial x^{2}} \frac{\partial u}{\partial t}-\frac{\partial}{\partial x}\left(\frac{\delta G}{\delta u}\right)\right\} \mathrm{d} x=\left[\frac{\partial}{\partial x} \frac{\partial u}{\partial t}-\frac{\delta G}{\delta u}\right]_{x=0}^{L}=0$.

The conservation of momentum (22) is also proved similarly,

$$
\begin{aligned}
\frac{d}{d t} I[u(\cdot, t)] & =\int_{0}^{L}\left(u \frac{\partial u}{\partial t}+\frac{\partial u}{\partial x} \frac{\partial^{2} u}{\partial x \partial t}\right) \mathrm{d} x \\
& =\int_{0}^{L}\left\{u\left(1-\frac{\partial^{2}}{\partial x^{2}}\right) \frac{\partial u}{\partial t}\right\} \mathrm{d} x+\left[u \frac{\partial^{2} u}{\partial x \partial t}\right]_{x=0}^{L} \\
& =-\int_{0}^{L} u \frac{\partial}{\partial x} \frac{\delta G}{\delta u} \mathrm{~d} x+\left[u \frac{\partial^{2} u}{\partial x \partial t}\right]_{x=0}^{L} \\
& =\int_{0}^{L} \frac{\partial u}{\partial x} \frac{\delta G}{\delta u} \mathrm{~d} x+\left[u\left(\frac{\partial^{2} u}{\partial x \partial t}-\frac{\delta G}{\delta u}\right)\right]_{x=0}^{L} \\
& =\int_{0}^{L} \frac{\partial}{\partial x} G \mathrm{~d} x+\left[u\left(\frac{\partial^{2} u}{\partial x \partial t}-\frac{\delta G}{\delta u}\right)\right]_{x=0}^{L} \\
& =\left[G+u\left(\frac{\partial^{2} u}{\partial x \partial t}-\frac{\delta G}{\delta u}\right)\right]_{x=0}^{L}=0
\end{aligned}
$$

where

$$
G(u) \stackrel{\text { def }}{=} \frac{1}{2} u^{2}+\frac{1}{6} u^{3}
$$


The conservation of energy (23) is proved as follows:

$$
\begin{aligned}
\frac{d}{d t} J[u(\cdot, t)] & =\int_{0}^{L} \frac{\delta G}{\delta u} \frac{\partial u}{\partial t} \mathrm{~d} x=\int_{0}^{L}\left\{\left(\frac{\delta G}{\delta u}-\frac{\partial^{2} u}{\partial x \partial t}\right) \frac{\partial u}{\partial t}+\frac{\partial^{2} u}{\partial x \partial t} \frac{\partial u}{\partial t}\right\} \mathrm{d} x \\
& =\int_{0}^{L}\left\{-\left(\frac{\partial^{2} u}{\partial x \partial t}-\frac{\delta G}{\delta u}\right) \frac{\partial}{\partial x}\left(\frac{\partial^{2} u}{\partial x \partial t}-\frac{\delta G}{\delta u}\right)+\frac{1}{2} \frac{\partial}{\partial x}\left(\frac{\partial u}{\partial t}\right)^{2}\right\} \mathrm{d} x \\
& =\frac{1}{2} \int_{0}^{L} \frac{\partial}{\partial x}\left\{-\left(\frac{\partial^{2} u}{\partial x \partial t}-\frac{\delta G}{\delta u}\right)^{2}+\left(\frac{\partial u}{\partial t}\right)^{2}\right\} \mathrm{d} x \\
& =\frac{1}{2}\left[-\left(\frac{\partial^{2} u}{\partial x \partial t}-\frac{\delta G}{\delta u}\right)^{2}+\left(\frac{\partial u}{\partial t}\right)^{2}\right]_{x=0}^{L}=0
\end{aligned}
$$

The main purpose of this subsection is to prove conservation properties of the proposed schemes corresponding to (21)-(23).

\subsubsection{Conservation properties of NM scheme}

The solutions $\boldsymbol{U}^{(n)}$ of the NM scheme (9) exhibit the following properties, i.e. discrete mass conservation property and discrete momentum conservation property:

$$
\begin{aligned}
M_{\mathrm{d}}\left[\boldsymbol{U}^{(n)}\right] & =M_{\mathrm{d}}\left[\boldsymbol{U}^{(0)}\right], \\
I_{\mathrm{d}}\left[\boldsymbol{U}^{(n)}\right] & =I_{\mathrm{d}}\left[\boldsymbol{U}^{(0)}\right],
\end{aligned}
$$

where

$$
\begin{aligned}
M_{\mathrm{d}}[\boldsymbol{f}] \stackrel{\text { def }}{=} \sum_{k=0}^{N-1} f_{k} \Delta x, \\
I_{\mathrm{d}}[\boldsymbol{f}] \stackrel{\text { def }}{=} \frac{1}{2} \sum_{k=0}^{N-1}\left\{\left(f_{k}\right)^{2}+\frac{\left(\delta_{k}^{+} f_{k}\right)^{2}+\left(\delta_{k}^{-} f_{k}\right)^{2}}{2}\right\} \Delta x .
\end{aligned}
$$

Here, $\delta_{k}^{+}$is a forward difference operator defined by $\delta_{k}^{+} f_{k}=\left(f_{k+1}-f_{k}\right) / \Delta x$ and $\delta_{k}^{-}$is a backward difference operator defined by $\delta_{k}^{-} f_{k}=\left(f_{k}-f_{k-1}\right) / \Delta x$.

The discrete mass conservation property (28) of the NM scheme is proved below. For simplicity, we denote $\boldsymbol{U}^{(n+1)}$ by $\boldsymbol{U}^{+}, \boldsymbol{U}^{(n)}$ by $\boldsymbol{U}$, and $\boldsymbol{U}^{(n-1)}$ by $\boldsymbol{U}^{-}$ in this subsection. We obtain

$$
\begin{aligned}
\frac{1}{\Delta t}\left(M_{\mathrm{d}}\left[\boldsymbol{U}^{+}\right]-M_{\mathrm{d}}[\boldsymbol{U}]\right) & =\sum_{k=0}^{N-1}\left(\frac{U_{k}^{+}-U_{k}}{\Delta t}\right) \Delta x \\
& =\sum_{k=0}^{N-1}\left\{\delta_{k}^{\langle 2\rangle} \delta_{n}^{+} U_{k}-\delta_{k}^{\langle 1\rangle}\left(\frac{\delta G_{\mathrm{d}}}{\delta\left(\boldsymbol{V}_{+}^{(n)}, \boldsymbol{V}_{-}^{(n)}\right)}\right)_{k}\right\} \Delta x \\
& =0
\end{aligned}
$$

by (121) and (122) (refer Appendix) because $\delta_{n}^{+} \boldsymbol{U}, \frac{\delta G_{\mathrm{d}}}{\delta\left(\boldsymbol{V}_{+}^{(n)}, \boldsymbol{V}_{-}^{(n)}\right)} \in \mathbf{R}^{(N)}$. 
The discrete momentum conservation property (29) of the NM scheme can be shown as follows:

$$
\begin{aligned}
& \frac{1}{\Delta t}\left(I_{\mathrm{d}}\left[\boldsymbol{U}^{+}\right]-I_{\mathrm{d}}[\boldsymbol{U}]\right) \\
= & \sum_{k=0}^{N-1}\left[\left(\frac{U_{k}^{+}+U_{k}}{2}\right)\left(\delta_{n}^{+} U_{k}\right)\right. \\
& \left.\quad+\frac{1}{2}\left\{\delta_{k}^{+}\left(\frac{U_{k}^{+}+U_{k}}{2}\right) \delta_{k}^{+}\left(\delta_{n}^{+} U_{k}\right)+\delta_{k}^{-}\left(\frac{U_{k}^{+}+U_{k}}{2}\right) \delta_{k}^{-}\left(\delta_{n}^{+} U_{k}\right)\right\}\right] \Delta x \\
= & \sum_{k=0}^{N-1}\left\{\left(\frac{U_{k}^{+}+U_{k}}{2}\right)\left(1-\delta_{k}^{\langle 2\rangle}\right)\left(\delta_{n}^{+} U_{k}\right)\right\} \Delta x \\
= & -\sum_{k=0}^{N-1}\left\{V_{k}^{(n)} \delta_{k}^{\langle 1\rangle}\left(\frac{\delta G_{\mathrm{d}}}{\delta\left(\boldsymbol{V}_{+}^{(n)}, \boldsymbol{V}_{-}^{(n)}\right)}\right)_{k}\right\} \Delta x \\
= & \sum_{k=0}^{N-1}\left(\delta_{k}^{\langle 1\rangle} V_{k}^{(n)}\right)\left(\frac{\delta G_{\mathrm{d}}}{\delta\left(\boldsymbol{V}_{+}^{(n)}, \boldsymbol{V}_{-}^{(n)}\right)}\right)_{k} \Delta x=\sum_{k=0}^{N-1} \delta_{k}^{\langle 1\rangle} G_{\mathrm{d}}\left(\boldsymbol{V}^{(n)}\right)_{k} \Delta x=0,
\end{aligned}
$$

where $V_{k}^{(n)} \stackrel{\text { def }}{=}\left(U_{k}^{(n+1)}+U_{k}^{(n)}\right) / 2$. The second, fourth, fifth, and sixth equalities are derived from (123), (124), (13), and (121), respectively.

\subsubsection{Conservation properties of NE scheme}

The solutions $\boldsymbol{U}^{(n)}$ of the NE scheme (14) exhibit the following properties, i.e. discrete mass conservation property and discrete energy conservation property:

$$
\begin{aligned}
M_{\mathrm{d}}\left[\boldsymbol{U}^{(n)}\right] & =M_{\mathrm{d}}\left[\boldsymbol{U}^{(0)}\right], \\
J_{\mathrm{d}}\left[\boldsymbol{U}^{(n)}\right] & =J_{\mathrm{d}}\left[\boldsymbol{U}^{(0)}\right],
\end{aligned}
$$

where

$$
J_{\mathrm{d}}[\boldsymbol{f}] \stackrel{\text { def }}{=} \sum_{k=0}^{N-1} G_{\mathrm{d}}(\boldsymbol{f})_{k} \Delta x .
$$

The discrete mass conservation property (34) of the NE scheme can be proved in a manner similar to the proof of (32) as follows:

$$
\begin{aligned}
& \frac{1}{\Delta t}\left(J_{\mathrm{d}}\left[\boldsymbol{U}^{+}\right]-J_{\mathrm{d}}[\boldsymbol{U}]\right) \\
& =\sum_{k=0}^{N-1}\left(\frac{\delta G_{\mathrm{d}}}{\delta\left(\boldsymbol{U}^{+}, \boldsymbol{U}\right)}\right)_{k} \delta_{n}^{+} U_{k} \Delta x \\
& =\sum_{k=0}^{N-1}\left[\left\{\left(\frac{\delta G_{\mathrm{d}}}{\delta\left(\boldsymbol{U}^{+}, \boldsymbol{U}\right)}\right)_{k}-\delta_{k}^{\langle 1\rangle} \delta_{n}^{+} U_{k}\right\} \delta_{n}^{+} U_{k}+\left(\delta_{k}^{\langle 1\rangle} \delta_{n}^{+} U_{k}\right) \delta_{n}^{+} U_{k}\right] \Delta x \\
& =-\sum_{k=0}^{N-1}\left[\left\{\left(\frac{\delta G_{\mathrm{d}}}{\delta\left(\boldsymbol{U}^{+}, \boldsymbol{U}\right)}\right)_{k}-\delta_{k}^{\langle 1\rangle} \delta_{n}^{+} U_{k}\right\} \delta_{k}^{\langle 1\rangle}\left\{\left(\frac{\delta G_{\mathrm{d}}}{\delta\left(\boldsymbol{U}^{+}, \boldsymbol{U}\right)}\right)_{k}-\delta_{k}^{\langle 1\rangle} \delta_{n}^{+} U_{k}\right\}\right] \Delta x \\
& =0
\end{aligned}
$$


where the third and fourth equalities are derived from (125).

\subsubsection{Conservation properties of LM scheme}

The solutions $\boldsymbol{U}^{(n)}$ of the LM scheme (15) exhibit the following properties, i.e. discrete mass conservation property and discrete momentum conservation property:

$$
\begin{aligned}
& M_{\mathrm{d}}\left[\boldsymbol{U}^{(n)}\right]= \begin{cases}M_{\mathrm{d}}\left[\boldsymbol{U}^{(1)}\right], & \text { for odd } n>0, \\
M_{\mathrm{d}}\left[\boldsymbol{U}^{(0)}\right], & \text { for even } n \geq 0,\end{cases} \\
& \tilde{I}_{\mathrm{d}}\left[\boldsymbol{U}^{(n+1)}, \boldsymbol{U}^{(n)}\right]=\tilde{I}_{\mathrm{d}}\left[\boldsymbol{U}^{(1)}, \boldsymbol{U}^{(0)}\right], \quad \text { for } n \geq 0,
\end{aligned}
$$

where

$$
\tilde{I}_{\mathrm{d}}[\boldsymbol{f}, \boldsymbol{g}] \stackrel{\text { def }}{=} \frac{1}{2} \sum_{k=0}^{N-1}\left\{f_{k} g_{k}+\frac{\left(\delta_{k}^{+} f_{k}\right)\left(\delta_{k}^{+} g_{k}\right)+\left(\delta_{k}^{-} f_{k}\right)\left(\delta_{k}^{-} g_{k}\right)}{2}\right\} \Delta x
$$

The discrete mass conservation property (38) of the LM scheme can be proved in a manner similar to the proof of $(32)$ as $\left(M_{\mathrm{d}}\left[\boldsymbol{U}^{(n+1)}\right]-M_{\mathrm{d}}\left[\boldsymbol{U}^{(n-1)}\right]\right) /(2 \Delta t)=0$. The discrete momentum conservation property (39) can be proved as follows:

$$
\begin{aligned}
& \frac{1}{\Delta t}\left(\tilde{I}_{\mathrm{d}}\left[\boldsymbol{U}^{+}, \boldsymbol{U}\right]-\tilde{I}_{\mathrm{d}}\left[\boldsymbol{U}, \boldsymbol{U}^{-}\right]\right) \\
= & \sum_{k=0}^{N-1}\left[U_{k}\left(\delta_{n}^{\langle 1\rangle} U_{k}\right)+\frac{1}{2}\left\{\left(\delta_{k}^{+} U_{k}\right)\left(\delta_{k}^{+} \delta_{n}^{\langle 1\rangle} U_{k}\right)+\left(\delta_{k}^{-} U_{k}\right)\left(\delta_{k}^{-} \delta_{n}^{\langle 1\rangle} U_{k}\right)\right\}\right] \Delta x \\
= & \sum_{k=0}^{N-1}\left\{U_{k}\left(1-\delta_{k}^{\langle 2\rangle}\right)\left(\delta_{n}^{\langle 1\rangle} U_{k}\right)\right\} \Delta x \\
= & -\sum_{k=0}^{N-1}\left\{V_{k}^{(n)} \delta_{k}^{\langle 1\rangle}\left(\frac{\delta G_{\mathrm{d}}}{\delta\left(\boldsymbol{U}_{+}^{(n)}, \boldsymbol{U}_{-}^{(n)}\right)}\right)_{k}\right\} \Delta x \\
= & \sum_{k=0}^{N-1}\left(\delta_{k}^{\langle 1\rangle} U_{k}^{(n)}\right)\left(\frac{\delta G_{\mathrm{d}}}{\delta\left(\boldsymbol{U}_{+}^{(n)}, \boldsymbol{U}_{-}^{(n)}\right)}\right)_{k} \Delta x=\sum_{k=0}^{N-1} \delta_{k}^{\langle 1\rangle} G_{\mathrm{d}}\left(\boldsymbol{U}^{(n)}\right)_{k} \Delta x=0 .
\end{aligned}
$$

\subsubsection{Conservation properties of LE scheme}

The solutions $\boldsymbol{U}^{(n)}$ of the LE scheme (17) exhibit the following properties, i.e. discrete mass conservation property and discrete energy conservation property:

$$
\begin{aligned}
& M_{\mathrm{d}}\left[\boldsymbol{U}^{(n)}\right]= \begin{cases}M_{\mathrm{d}}\left[\boldsymbol{U}^{(1)}\right], & \text { for odd } n>0, \\
M_{\mathrm{d}}\left[\boldsymbol{U}^{(0)}\right], & \text { for even } n \geq 0,\end{cases} \\
& \tilde{J}_{\mathrm{d}}\left[\boldsymbol{U}^{(n+1)}, \boldsymbol{U}^{(n)}\right]=\tilde{J}_{\mathrm{d}}\left[\boldsymbol{U}^{(1)}, \boldsymbol{U}^{(0)}\right], \quad \text { for } n \geq 0 \text {, }
\end{aligned}
$$

where

$$
\tilde{J}_{\mathrm{d}}[\boldsymbol{f}, \boldsymbol{g}] \stackrel{\text { def }}{=} \sum_{k=0}^{N-1} \tilde{G}_{\mathrm{d}}(\boldsymbol{f}, \boldsymbol{g})_{k} \Delta x
$$


The discrete mass conservation property (42) of the LE scheme can be proved in a manner similar to the proof of (32). The discrete momentum conservation property (43) can be proved as follows:

$$
\begin{aligned}
& \frac{1}{\Delta t}\left(\tilde{J}_{\mathrm{d}}\left[\boldsymbol{U}^{+}, \boldsymbol{U}\right]-\tilde{J}_{\mathrm{d}}\left[\boldsymbol{U}, \boldsymbol{U}^{-}\right]\right) \\
& =\sum_{k=0}^{N-1}\left(\frac{\delta \tilde{G}_{\mathrm{d}}}{\delta\left(\boldsymbol{U}^{+}, \boldsymbol{U}, \boldsymbol{U}^{-}\right)}\right)_{k} \delta_{n}^{\langle 1\rangle} U_{k} \Delta x \\
& =\sum_{k=0}^{N-1}\left[\left\{\left(\frac{\delta \tilde{G}_{\mathrm{d}}}{\delta\left(\boldsymbol{U}^{+}, \boldsymbol{U}, \boldsymbol{U}^{-}\right)}\right)_{k}-\delta_{k}^{\langle 1\rangle} \delta_{n}^{\langle 1\rangle} U_{k}\right\} \delta_{n}^{\langle 1\rangle} U_{k}+\left(\delta_{k}^{\langle 1\rangle} \delta_{n}^{\langle 1\rangle} U_{k}\right) \delta_{n}^{\langle 1\rangle} U_{k}\right] \Delta x \\
& =-\sum_{k=0}^{N-1}\left[\left\{\left(\frac{\delta \tilde{G}_{\mathrm{d}}}{\delta\left(\boldsymbol{U}^{+}, \boldsymbol{U}, \boldsymbol{U}^{-}\right)}\right)_{k}-\delta_{k}^{\langle 1\rangle} \delta_{n}^{\langle 1\rangle} U_{k}\right\}\right. \\
& \left.\quad \times \delta_{k}^{\langle 1\rangle}\left\{\left(\frac{\delta \tilde{G}_{\mathrm{d}}}{\delta\left(\boldsymbol{U}^{+}, \boldsymbol{U}^{-} \boldsymbol{U}^{-}\right)}\right)_{k}-\delta_{k}^{\langle 1\rangle} \delta_{n}^{\langle 1\rangle} U_{k}\right\}\right] \Delta x=0,
\end{aligned}
$$

where the third and fourth equalities are derived from (125).

\section{Solution stability, existence, and convergence of NM scheme}

\subsection{Solution stability of NM scheme}

The purpose of this subsection is to prove that if the numerical solutions of the NM scheme exist, they are bounded by the maximum norm. The proof of the property consists of a lemma-discrete Sobolev lemma - which shows that the maximum norm of a discrete function is bounded when its discrete Sobolev norm is bounded.

Lemma 1 (Discrete Sobolev lemma).

$$
\max _{k \in \mathbf{Z}}\left|f_{k}\right| \leq 2 \max \left(\frac{1}{\sqrt{L}}, \sqrt{L}\right)\|\boldsymbol{f}\|_{\mathrm{d}-(1,2)},
$$

where

$$
\|\boldsymbol{f}\|_{\mathrm{d}-(1,2)} \stackrel{\text { def }}{=}\left[\sum_{k=0}^{N-1}\left\{\left(f_{k}\right)^{2}+\frac{\left(\delta_{k}^{+} f_{k}\right)^{2}+\left(\delta_{k}^{-} f_{k}\right)^{2}}{2}\right\} \Delta x\right]^{1 / 2} .
$$

Proof. There exists a simple proof for this lemma in $[13, \S 8.6]$.

Applying this lemma to (29), we obtain the following inequality:

THEOREM 2.

$$
\max _{k \in \mathbf{Z}}\left|U_{k}^{(n)}\right| \leq 2 \sqrt{2} \max \left(\frac{1}{\sqrt{L}}, \sqrt{L}\right) \sqrt{I_{\mathrm{d}}\left[\boldsymbol{U}^{(0)}\right]} .
$$

This inequality implies that the NM scheme (9) is stable for any time step $n$ if the numerical solutions of the scheme exist. 


\subsection{Existence of solutions of NM scheme}

In this subsection, using the fixed-point theorem for contraction mapping, we show that the NM scheme (9) has unique solutions under some conditions of $\Delta t$ and $\Delta x$. For the scheme (9), we define a mapping $\mathcal{T}_{U^{(n)}}: \mathbf{R}^{N} \rightarrow \mathbf{R}^{N}$ as

$$
\frac{2}{\Delta t}\left(I-D_{2}\right)\left(\mathcal{T}_{U^{(n)}} \boldsymbol{v}-\tilde{\boldsymbol{U}}^{(n)}\right) \stackrel{\text { def }}{=} D_{1} \boldsymbol{A}(\boldsymbol{v})
$$

where $I$ is the identity matrix of order $N ; D_{2}$ is the second difference operator matrix of order $N$ under the boundary condition (7),

$$
D_{2} \stackrel{\text { def }}{=} \frac{1}{(\Delta x)^{2}}\left(\begin{array}{ccccccc}
-2 & 1 & & & & 1 \\
1 & -2 & 1 & & 0 & \\
& 1 & -2 & 1 & & \\
& & \ddots & \ddots & \ddots & \\
& 0 & & 1 & -2 & 1 \\
1 & & & & 1 & -2
\end{array}\right)
$$

$\tilde{\boldsymbol{U}}^{(n)}$ is an $\mathbf{R}^{N}$ vector defined as $\tilde{U}_{k}^{(n)} \stackrel{\text { def }}{=} U_{k}^{(n)}$ for $k=0,1, \ldots, N-1 ; D_{1}$ is the first difference operator matrix,

$$
D_{1} \stackrel{\text { def }}{=} \frac{1}{2 \Delta x}\left(\begin{array}{ccccccc}
0 & 1 & & & & -1 \\
-1 & 0 & 1 & & 0 & \\
& -1 & 0 & 1 & & \\
& & \ddots & \ddots & \ddots & \\
& 0 & & -1 & 0 & 1 \\
1 & & & & -1 & 0
\end{array}\right)
$$

and

$$
\begin{aligned}
& \{\boldsymbol{A}(\boldsymbol{v})\}_{k} \\
& \stackrel{\text { def }}{=} \begin{cases}\left(\frac{v_{1}+v_{N-1}}{2}\right)+\frac{\left(v_{1}\right)^{2}+\left(v_{1}\right)\left(v_{N-1}\right)+\left(v_{N-1}\right)^{2}}{6}, & \text { for } k=0, \\
\left(\frac{v_{k+1}+v_{k-1}}{2}\right)+\frac{\left(v_{k+1}\right)^{2}+\left(v_{k+1}\right)\left(v_{k-1}\right)+\left(v_{k-1}\right)^{2}}{6}, & \text { for } k=1,2, \ldots, N-2, \\
\left(\frac{v_{0}+v_{N-2}}{2}\right)+\frac{\left(v_{0}\right)^{2}+\left(v_{0}\right)\left(v_{N-2}\right)+\left(v_{N-2}\right)^{2}}{6}, & \text { for } k=N-1 .\end{cases}
\end{aligned}
$$

Note that the operator $\left(I-D_{2}\right)$ is a nonsingular matrix. The eigenvalues of $\left(I-D_{2}\right)$ are

$$
1-\frac{2}{(\Delta x)^{2}}\left\{\cos \left(\frac{2 k \pi}{N}\right)-1\right\}, \quad k=0,1, \ldots, N-1 .
$$


It is clear that all eigenvalues are larger than 1, implying that the operator is a nonsingular matrix.

When $\boldsymbol{v}$ is assumed to be $\left(\boldsymbol{U}^{(n+1)}+\boldsymbol{U}^{(n)}\right) / 2$, the equation $\mathcal{T}_{U^{(n)}} \boldsymbol{v}=\boldsymbol{v}$ is essentially no different from the NM scheme (9). Therefore, we prove the existence of solution of the NM scheme using the fixed-point theorem for the mapping $\mathcal{T}_{U^{(n)}}$.

Theorem 3 (Local solution existence and uniqueness). If

$$
\Delta t \leq \frac{2 \Delta x}{1+2 \mathcal{K}^{(n)}}
$$

then the mapping $\mathcal{T}_{U^{(n)}}$ has a unique solution in a closed ball $\mathcal{B}^{(n)}$, where

$$
\begin{aligned}
&\|\boldsymbol{f}\| \stackrel{\text { def }}{=}\left\{\sum_{k=0}^{N-1}\left(f_{k}\right)^{2}\right\}^{1 / 2}, \\
& \mathcal{K}^{(n)} \stackrel{\text { def }}{=}\left\|\tilde{\boldsymbol{U}}^{(n)}\right\|, \\
& \mathcal{B}^{(n)} \stackrel{\text { def }}{=}\left\{\boldsymbol{f} \in \mathbf{R}^{N} \mid\|\boldsymbol{f}\| \leq 2 \mathcal{K}^{(n)}\right\} .
\end{aligned}
$$

Proof. We assume that $\boldsymbol{v} \in \mathcal{B}^{(n)}$ and $\boldsymbol{v}^{\prime} \in \mathcal{B}^{(n)}$ in this proof. Using the mapping $\mathcal{T}_{U^{(n)}}$, we obtain

$$
\begin{aligned}
\left\|\mathcal{T}_{U^{(n)}} \boldsymbol{v}-\mathcal{T}_{U^{(n)}} \boldsymbol{v}^{\prime}\right\| & \leq \frac{\Delta t}{2}\left\|\left(I-D_{2}\right)^{-1}\right\|\left\|D_{1}\right\|\left\|\boldsymbol{A}(\boldsymbol{v})-\boldsymbol{A}\left(\boldsymbol{v}^{\prime}\right)\right\| \\
& \leq \frac{\Delta t}{2 \Delta x}\left\|\boldsymbol{A}(\boldsymbol{v})-\boldsymbol{A}\left(\boldsymbol{v}^{\prime}\right)\right\|,
\end{aligned}
$$

where the norm of a matrix is defined as an operator norm. In order to evaluate the abovementioned matrix norm, using the eigenvalues of $\left(I-D_{2}\right)(53)$, we determine $\left\|\left(I-D_{2}\right)^{-1}\right\|=1$. Using $D_{1}$, we can show that $\left\|D_{1}\right\| \leq \frac{1}{\Delta x}$ because

$$
\left|\lambda_{k}\right|=\frac{\sin \left(\frac{2 k \pi}{N}\right)}{\Delta x}, \quad k=0,1, \ldots, N-1,
$$

for the eigenvalues $\lambda_{k}$ of $D_{1}$. On the right-hand side of the inequality (58),

$$
\begin{aligned}
\left\|\boldsymbol{A}(\boldsymbol{v})-\boldsymbol{A}\left(\boldsymbol{v}^{\prime}\right)\right\| \leq & \frac{1}{2}\left(\left\|\boldsymbol{v}_{+}-\boldsymbol{v}_{+}^{\prime}\right\|+\left\|\boldsymbol{v}_{-}-\boldsymbol{v}_{-}^{\prime}\right\|\right) \\
& +\frac{1}{6}\left(\left\|\boldsymbol{w}_{+}-\boldsymbol{w}_{+}^{\prime}\right\|+\left\|\boldsymbol{w}-\boldsymbol{w}^{\prime}\right\|+\left\|\boldsymbol{w}_{-}-\boldsymbol{w}_{-}^{\prime}\right\|\right)
\end{aligned}
$$

where

$$
\begin{aligned}
& \left(\boldsymbol{v}_{+}\right)_{k} \stackrel{\text { def }}{=} \begin{cases}v_{k+1}, & k=0,1, \ldots, N-2, \\
v_{0}, & k=N-1,\end{cases} \\
& \left(\boldsymbol{v}_{-}\right)_{k} \stackrel{\text { def }}{=} \begin{cases}v_{N-1}, & k=0, \\
v_{k-1}, & k=1,2, \ldots, N-1,\end{cases}
\end{aligned}
$$




$$
\begin{gathered}
\left(\boldsymbol{w}_{+}\right)_{k} \stackrel{\text { def }}{=}\left\{\left(\boldsymbol{v}_{+}\right)_{k}\right\}^{2}, \quad k=0,1, \ldots, N-1, \\
(\boldsymbol{w})_{k} \stackrel{\text { def }}{=}\left(\boldsymbol{v}_{+}\right)_{k}\left(\boldsymbol{v}_{-}\right)_{k}, \quad k=0,1, \ldots, N-1, \\
\left(\boldsymbol{w}_{-}\right)_{k} \stackrel{\text { def }}{=}\left\{\left(\boldsymbol{v}_{-}\right)_{k}\right\}^{2}, \quad k=0,1, \ldots, N-1 .
\end{gathered}
$$

Further, $\boldsymbol{v}_{+}^{\prime}, \boldsymbol{v}_{-}^{\prime}, \boldsymbol{w}_{+}^{\prime}, \boldsymbol{w}^{\prime}$, and $\boldsymbol{w}_{-}^{\prime}$ are defined in a similar manner. First,

$$
\left\|v_{+}-v_{+}^{\prime}\right\|=\left\|v_{-}-v_{-}^{\prime}\right\|=\left\|v-v^{\prime}\right\|
$$

is obvious. Next,

$$
\begin{aligned}
\left\|\boldsymbol{w}_{+}-\boldsymbol{w}_{+}^{\prime}\right\|^{2} & =\sum_{k=0}^{N-1}\left(v_{k}+v_{k}^{\prime}\right)^{2}\left(v_{k}-v_{k}^{\prime}\right)^{2} \\
& \leq \max _{0 \leq k \leq N-1}\left(v_{k}+v_{k}^{\prime}\right)^{2}\left\|\boldsymbol{v}-\boldsymbol{v}^{\prime}\right\|^{2} \\
& \leq 2 \max _{0 \leq k \leq N-1}\left\{\left(v_{k}\right)^{2}+\left(v_{k}^{\prime}\right)^{2}\right\}\left\|\boldsymbol{v}-\boldsymbol{v}^{\prime}\right\|^{2} \\
& \leq 2\left\{\|\boldsymbol{v}\|^{2}+\left\|\boldsymbol{v}^{\prime}\right\|^{2}\right\}\left\|\boldsymbol{v}-\boldsymbol{v}^{\prime}\right\|^{2} \\
& \leq 16\left(\mathcal{K}^{(n)}\right)^{2}\left\|\boldsymbol{v}-\boldsymbol{v}^{\prime}\right\|^{2} .
\end{aligned}
$$

Similarly, $\left\|\boldsymbol{w}_{-}-\boldsymbol{w}_{-}^{\prime}\right\| \leq 4 \mathcal{K}^{(n)}\left\|\boldsymbol{v}-\boldsymbol{v}^{\prime}\right\|$ can be obtained. Further, $\boldsymbol{w}-\boldsymbol{w}^{\prime}=$ $\left(z_{1}+z_{2}\right) / 2$, where

$$
\begin{aligned}
& \left(\boldsymbol{z}_{1}\right)_{k} \stackrel{\text { def }}{=}\left\{\left(\boldsymbol{v}_{+}\right)_{k}+\left(\boldsymbol{v}_{+}^{\prime}\right)_{k}\right\}\left\{\left(\boldsymbol{v}_{-}\right)_{k}-\left(\boldsymbol{v}_{-}^{\prime}\right)_{k}\right\}, \\
& \left(\boldsymbol{z}_{2}\right)_{k} \stackrel{\text { def }}{=}\left\{\left(\boldsymbol{v}_{+}\right)_{k}-\left(\boldsymbol{v}_{+}^{\prime}\right)_{k}\right\}\left\{\left(\boldsymbol{v}_{-}\right)_{k}+\left(\boldsymbol{v}_{-}^{\prime}\right)_{k}\right\} .
\end{aligned}
$$

Since

$$
\left\|\boldsymbol{z}_{1}\right\|^{2} \leq \max _{0 \leq k \leq N-1}\left(v_{k}+v_{k}^{\prime}\right)^{2}\left\|\boldsymbol{v}-\boldsymbol{v}^{\prime}\right\|^{2} \leq 16\left(\mathcal{K}^{(n)}\right)^{2}\left\|\boldsymbol{v}-\boldsymbol{v}^{\prime}\right\|^{2}
$$

and $\left\|\boldsymbol{z}_{2}\right\|^{2} \leq 16\left(\mathcal{K}^{(n)}\right)^{2}\left\|\boldsymbol{v}-\boldsymbol{v}^{\prime}\right\|^{2}$, we obtain $\left\|\boldsymbol{w}-\boldsymbol{w}^{\prime}\right\| \leq 4 \mathcal{K}^{(n)}\left\|\boldsymbol{v}-\boldsymbol{v}^{\prime}\right\|$. Hence, we obtain

$$
\left\|\boldsymbol{A}(\boldsymbol{v})-\boldsymbol{A}\left(\boldsymbol{v}^{\prime}\right)\right\| \leq\left(1+2 \mathcal{K}^{(n)}\right)\left\|\boldsymbol{v}-\boldsymbol{v}^{\prime}\right\|
$$

and

$$
\left\|\mathcal{T}_{U^{(n)}} \boldsymbol{v}-\mathcal{T}_{U^{(n)}} \boldsymbol{v}^{\prime}\right\| \leq \frac{\Delta t}{2 \Delta x}\left(1+2 \mathcal{K}^{(n)}\right)\left\|\boldsymbol{v}-\boldsymbol{v}^{\prime}\right\| .
$$

This implies that the mapping $\mathcal{T}_{U^{(n)}}$ contracts when $\Delta t$ satisfies

$$
\frac{\Delta t}{2 \Delta x}\left(1+2 \mathcal{K}^{(n)}\right)<1 \Longleftrightarrow \Delta t<\frac{2 \Delta x}{1+2 \mathcal{K}^{(n)}}
$$


Substituting $\mathcal{K}^{(n)}$ in Theorem 3, we obtain

$$
\left(\mathcal{K}^{(n)}\right)^{2}=\frac{1}{\Delta x}\left\{\sum_{k=0}^{N-1}\left(U_{k}^{(n)}\right)^{2} \Delta x\right\} \leq \frac{1}{\Delta x}\left\|\boldsymbol{U}^{(n)}\right\|_{\mathrm{d}-(1,2)}^{2}=\frac{2}{\Delta x} I_{\mathrm{d}}\left[\boldsymbol{U}^{(n)}\right]=\frac{2}{\Delta x} I_{\mathrm{d}}\left[\boldsymbol{U}^{(0)}\right]
$$

because $\boldsymbol{U}^{(n)}$ are the solutions of the NM scheme. Using this inequality, we obtain the following corollary.

Corollary 4 (Global solution existence and uniqueness). If

$$
\Delta t \leq \frac{\Delta x^{\frac{3}{2}}}{\left(2 I_{\mathrm{d}}\left[\boldsymbol{U}^{(0)}\right]\right)^{\frac{1}{2}}+\frac{\Delta x^{\frac{1}{2}}}{2}}
$$

the NM scheme (9) has unique numerical solutions $\boldsymbol{U}^{(n)}$ for any $n \geq 0$.

\subsection{Error estimate for NM scheme}

TheOrem 5. We assume that $0 \leq t \leq T$ with $T<\infty$ and $N=L / \Delta x \geq 12$. When the $R L W$ equation solution $u(x, \cdot)$ satisfies the condition

$$
\left|\frac{\partial^{l}}{\partial x^{l}} \frac{\partial^{m}}{\partial t^{m}} u(x, t)\right|<\infty, \quad \text { for } x \in \mathbf{R}, 0 \leq t \leq T, 0 \leq l \leq 5,0 \leq m \leq 3,
$$

and the time mesh size $\Delta t$ satisfies the condition

$$
\Delta t<\frac{2}{3 \lambda}
$$

the difference between the NM scheme solution $U_{k}^{(n)}$ and the PDE solution $u(x, t)$ is evaluated as follows:

$$
\max _{k \in \mathbf{Z}}\left|U_{k}^{(n)}-u(k \Delta x, n \Delta t)\right| \leq \sqrt{6 T} \max (1, L) E_{0} e^{\frac{3}{4} \lambda T}, \quad \text { for } n \leq \frac{T}{\Delta t},
$$

where

$$
\begin{gathered}
\lambda \stackrel{\text { def }}{=} 2^{9 / 8} \max \left((4 / L)^{1 / 4}, 1\right)\left(I[u(\cdot, 0)]+\frac{7}{4} L \mathcal{L}^{2} \Delta x^{2}\right)^{1 / 2}+1 \\
E_{0} \stackrel{\text { def }}{=}\left(\Delta x^{2}+\Delta t^{2}\right)\left(1+\Delta x^{2}+\Delta t^{2}+\Delta x^{4}+\Delta t^{4}\right)\left((49 / 32) \mathcal{L}+(14245 / 3456) \mathcal{L}^{2}\right), \\
\mathcal{L} \stackrel{\text { def }}{=} \sup _{0 \leq t \leq T, x \in \mathbf{R}, 0 \leq m \leq 3, l \in a(m)}\left\{\left|\frac{\partial^{l}}{\partial x^{l}} \frac{\partial^{m}}{\partial t^{m}} u(x, t)\right|\right\} \\
a(m) \stackrel{\text { def }}{=} \begin{cases}\{0,1,2,3,4,5\}, & m=0 \\
\{3,4\}, & m=1 \\
\{0,1,2,3,4,5\}, & m=2 \\
\{0,1,2,3,4\}, & m=3 .\end{cases}
\end{gathered}
$$


We require a small lemma to obtain the proof of this theorem.

LEMma 6. For any function $f(x) \in C^{3}[-\Delta x, L]$, which satisfies the periodic boundary conditions,

$$
f(x)=f(x+L), \quad \text { for } x \in[-\Delta x, 0],
$$

the estimate

$$
\left|I_{\mathrm{d}}[\boldsymbol{f}]-I[f]\right| \leq \frac{7}{4} L \mathcal{C}^{2} \Delta x^{2}
$$

is obtained, where

$$
\begin{aligned}
(\boldsymbol{f})_{k} & \stackrel{\text { def }}{=} f(k \Delta x), \\
\mathcal{C} & \stackrel{\text { def }}{=} \sup _{x \in[0, L], 0 \leq l \leq 3}\left\{\left|\frac{\partial^{l}}{\partial x^{l}} f(x)\right|\right\} .
\end{aligned}
$$

Proof.

$$
\begin{aligned}
\left|I_{\mathrm{d}}[\boldsymbol{f}]-I[f]\right| \leq & \left|\sum_{k=0}^{N-1} H_{\mathrm{d}}(\boldsymbol{f})_{k} \Delta x-\sum_{k=0}^{N-1} H(f)_{k} \Delta x\right| \\
& +\left|\sum_{k=0}^{N-1} H(f)_{k} \Delta x-\int_{0}^{L} H(f) \mathrm{d} x\right|
\end{aligned}
$$

where

$$
\begin{aligned}
H_{\mathrm{d}}(\boldsymbol{f})_{k} & \stackrel{\text { def }}{=} \frac{1}{2}\left\{\left(f_{k}\right)^{2}+\frac{\left(\delta_{k}^{+} f_{k}\right)^{2}+\left(\delta_{k}^{-} f_{k}\right)^{2}}{2}\right\}, \\
H(f) & \stackrel{\text { def }}{=} \frac{1}{2}\left\{f^{2}+\left(\frac{\partial f}{\partial x}\right)^{2}\right\}, \\
H(f)_{k} & \left.\stackrel{\text { def }}{=} H(f)\right|_{x=k \Delta x} .
\end{aligned}
$$

The first term on the right-hand side of (87) is evaluated as

$$
\left|\sum_{k=0}^{N-1} H_{\mathrm{d}}(\boldsymbol{f})_{k} \Delta x-\sum_{k=0}^{N-1} H(f)_{k} \Delta x\right| \leq \frac{5}{4} L \mathcal{C}^{2} \Delta x^{2}
$$

by the iterative applications of the Taylor expansion. The second term is evaluated as

$$
\left|\sum_{k=0}^{N-1} H(f)_{k} \Delta x-\int_{0}^{L} H(f) \mathrm{d} x\right| \leq \frac{\Delta x^{2}}{8} \int_{0}^{L}\left|\frac{\partial^{2}}{\partial x^{2}} H(f)\right| \mathrm{d} x \leq \frac{1}{2} L \mathcal{C}^{2} \Delta x^{2}
$$

by using the Euler-Maclaurin summation formula since $H(f) \in C^{2}[0, L]$. 
Proof of Error estimate. The NM scheme (9) is identical to

$$
0=\left(1-\delta_{k}^{\langle 2\rangle}\right) \delta_{n}^{+} U_{k}^{(n)}+\delta_{k}^{\langle 1\rangle} s_{k}^{\langle 1\rangle}\left(\frac{U_{k}^{(n+1)}+U_{k}^{(n)}}{2}\right)+\frac{1}{2} \delta_{k}^{\langle 1\rangle} \xi\left(\frac{\boldsymbol{U}^{(n+1)}+\boldsymbol{U}^{(n)}}{2}\right)_{k},
$$

where

$$
\begin{aligned}
& s_{k}^{\langle 1\rangle} f_{k} \stackrel{\text { def }}{=}\left(f_{k+1}+f_{k-1}\right) / 2, \\
& \xi(\boldsymbol{f})_{k} \stackrel{\text { def }}{=}\left\{\left(f_{k+1}\right)^{2}+\left(f_{k+1}\right)\left(f_{k-1}\right)+\left(f_{k-1}\right)^{2}\right\} / 3 .
\end{aligned}
$$

By the iterative applications of the Taylor expansion, we obtain the following equation as to the solution of the RLW equation as follows:

$$
0=\left(1-\delta_{k}^{\langle 2\rangle}\right) \delta_{n}^{+} u_{k}^{(n)}+\delta_{k}^{\langle 1\rangle} s_{k}^{\langle 1\rangle}\left(\frac{u_{k}^{(n+1)}+u_{k}^{(n)}}{2}\right)+\frac{1}{2} \delta_{k}^{\langle 1\rangle} \xi\left(\frac{\boldsymbol{u}^{(n+1)}+\boldsymbol{u}^{(n)}}{2}\right)_{k}+E_{k}^{(n)}
$$

where $E_{k}^{(n)}$ is the summation of residual factors using the Taylor expansion,

$$
\begin{aligned}
\left(\boldsymbol{u}^{(n)}\right)_{k} & \stackrel{\text { def }}{=} u_{k}^{(n)}, \\
u_{k}^{(n)} & \stackrel{\text { def }}{=} u(k \Delta x, n \Delta t), \\
\left|E_{k}^{(n)}\right| & \leq E_{0} .
\end{aligned}
$$

For simplicity, we denote error in the numerical solutions by

$$
e_{k}^{(n)} \stackrel{\text { def }}{=} U_{k}^{(n)}-u_{k}^{(n)}
$$

Subtracting (96) from (93), we obtain

$$
\begin{aligned}
0= & \left(1-\delta_{k}^{\langle 2\rangle}\right) \delta_{n}^{+} e_{k}^{(n)}+\delta_{k}^{\langle 1\rangle} s_{k}^{\langle 1\rangle} \tilde{e}_{k}^{(n)} \\
& +\frac{1}{2} \delta_{k}^{\langle 1\rangle}\left\{\xi\left(\frac{\boldsymbol{U}^{(n+1)}+\boldsymbol{U}^{(n)}}{2}\right)_{k}-\xi\left(\frac{\boldsymbol{u}^{(n+1)}+\boldsymbol{u}^{(n)}}{2}\right)_{k}\right\}-E_{k}^{(n)} \\
= & \left(1-\delta_{k}^{\langle 2\rangle}\right) \delta_{n}^{+} e_{k}^{(n)}+\delta_{k}^{\langle 1\rangle} s_{k}^{\langle 1\rangle} \tilde{e}_{k}^{(n)} \\
& +\frac{1}{3} \delta_{k}^{\langle 1\rangle}\left\{2\left(s_{k}^{\langle 1\rangle} \mu_{k}^{(n)}\right)\left(s_{k}^{\langle 1\rangle} \tilde{e}_{k}^{(n)}\right)+s_{k}^{\langle 1\rangle}\left(\mu_{k}^{(n)} \tilde{e}_{k}^{(n)}\right)\right\}-E_{k}^{(n)}
\end{aligned}
$$

where

$$
\begin{aligned}
\tilde{e}_{k}^{(n)} \stackrel{\text { def }}{=} \frac{e_{k}^{(n+1)}+e_{k}^{(n)}}{2} \\
\mu_{k}^{(n)} \stackrel{\text { def }}{=} \frac{1}{4}\left(U_{k}^{(n+1)}+U_{k}^{(n)}+u_{k}^{(n+1)}+u_{k}^{(n)}\right) .
\end{aligned}
$$


Multiplying the equation by $2 \tilde{e}_{k}^{(n)}$ and operating $\sum_{k=0}^{N-1} \Delta x$, we obtain

$$
\begin{aligned}
0= & \frac{1}{\Delta t}\left(\left\|\boldsymbol{e}^{(n+1)}\right\|_{\mathrm{d}-(1,2)}^{2}-\|\boldsymbol{e}\|_{\mathrm{d}-(1,2)}^{2}\right)+\frac{1}{6} \sum_{k=0}^{N-1} \frac{\mu_{k+2}^{(n)}-\mu_{k-2}^{(n)}}{4 \Delta x}\left(\tilde{e}_{k}^{(n)}\right)^{2} 4 \Delta x \\
& +\frac{1}{6} \sum_{k=0}^{N-1} \frac{\mu_{k+1}^{(n)}-\mu_{k-1}^{(n)}}{2 \Delta x}\left(\tilde{e}_{k+1}^{(n)} \tilde{e}_{k-1}^{(n)}\right) 2 \Delta x-2 \sum_{k=0}^{N-1} \tilde{e}_{k}^{(n)} E_{k}^{(n)} \Delta x .
\end{aligned}
$$

Applying the Schwartz inequality, we can evaluate the second term o the right-hand side of this equation as

$$
\begin{aligned}
& \frac{1}{6} \sum_{k=0}^{N-1} \frac{\mu_{k+2}^{(n)}-\mu_{k-2}^{(n)}}{4 \Delta x}\left(\tilde{e}_{k}^{(n)}\right)^{2} 4 \Delta x \\
& =\frac{1}{6} \sum_{k=0}^{N-1}\left(\delta_{k}^{+} \mu_{k+1}^{(n)}+\delta_{k}^{+} \mu_{k}^{(n)}+\delta_{k}^{-} \mu_{k}^{(n)}+\delta_{k}^{-} \mu_{k-1}^{(n)}\right)\left(\tilde{e}_{k}^{(n)}\right)^{2} \Delta x \\
& \leq \frac{2}{3}\left\{\sum_{k=0}^{N-1}\left(\delta_{k}^{+} \mu_{k}^{(n)}\right)^{2} \Delta x\right\}^{1 / 2}\left\{\sum_{k=0}^{N-1}\left(\tilde{e}_{k}^{(n)}\right)^{4} \Delta x\right\}^{1 / 2} \\
& \leq \frac{2}{3}\left\|\boldsymbol{\mu}^{(n)}\right\|_{\mathrm{d}-(1,2)}\left\|\tilde{\boldsymbol{e}}^{(n)}\right\|_{\mathrm{d}-(0,4)}^{2},
\end{aligned}
$$

where

$$
\|\boldsymbol{f}\|_{\mathrm{d}-(0,4)} \stackrel{\text { def }}{=}\left\{\sum_{k=0}^{N-1}\left(f_{k}\right)^{4} \Delta x\right\}^{1 / 4} .
$$

Similarly, evaluating the third term on the right-hand side of (104), we get

$$
\frac{1}{6} \sum_{k=0}^{N-1} \frac{\mu_{k+1}^{(n)}-\mu_{k-1}^{(n)}}{2 \Delta x}\left(\tilde{e}_{k+1}^{(n)} \tilde{e}_{k-1}^{(n)}\right) 2 \Delta x \leq \frac{1}{3}\left\|\boldsymbol{\mu}^{(n)}\right\|_{\mathrm{d}-(1,2)}\left\|\tilde{\boldsymbol{e}}^{(n)}\right\|_{\mathrm{d}-(0,4)}^{2} .
$$

The last term on the right-hand side of (104) is evaluated as

$$
\begin{aligned}
\left|2 \sum_{k=0}^{N-1} \tilde{e}_{k}^{(n)} E_{k}^{(n)} \Delta x\right| & \leq \sum_{k=0}^{N-1}\left\{\left(\tilde{e}_{k}^{(n)}\right)^{2}+\left(E_{k}^{(n)}\right)^{2}\right\} \Delta x \\
& \leq\left\|\tilde{\boldsymbol{e}}^{(n)}\right\|_{\mathrm{d}-(1,2)}+L\left(E_{0}\right)^{2} .
\end{aligned}
$$

According to the discrete Gagliardo-Nirenberg inequality given by Matsuo et al. [15, §5.2],

$$
\|\boldsymbol{f}\|_{\mathrm{d}-(0,4)} \leq 32^{1 / 8} \max \left((4 / L)^{1 / 4}, 1\right)\|\boldsymbol{f}\|_{\mathrm{d}-(1,2)}
$$


when $N \geq 12$. Using Lemma $6,\left\|\boldsymbol{\mu}^{(n)}\right\|_{\mathrm{d}-(1,2)}$ can be evaluated as

$$
\begin{aligned}
& \left\|\boldsymbol{\mu}^{(n)}\right\|_{\mathrm{d}-(1,2)} \\
& \leq \frac{\sqrt{2}}{4}\left\{\left(I_{\mathrm{d}}\left[\boldsymbol{U}^{(n+1)}\right]\right)^{1 / 2}+\left(I_{\mathrm{d}}\left[\boldsymbol{U}^{(n)}\right]\right)^{1 / 2}+\left(I_{\mathrm{d}}\left[\boldsymbol{u}^{(n+1)}\right]\right)^{1 / 2}+\left(I_{\mathrm{d}}\left[\boldsymbol{u}^{(n)}\right]\right)^{1 / 2}\right\} \\
& =\frac{\sqrt{2}}{4}\left\{2\left(I_{\mathrm{d}}\left[\boldsymbol{U}^{(0)}\right]\right)^{1 / 2}+\left(I_{\mathrm{d}}\left[\boldsymbol{u}^{(n+1)}\right]\right)^{1 / 2}+\left(I_{\mathrm{d}}\left[\boldsymbol{u}^{(n)}\right]\right)^{1 / 2}\right\} \\
& =\frac{\sqrt{2}}{4}\left\{2\left(I_{\mathrm{d}}\left[\boldsymbol{u}^{(0)}\right]\right)^{1 / 2}+\left(I_{\mathrm{d}}\left[\boldsymbol{u}^{(n+1)}\right]\right)^{1 / 2}+\left(I_{\mathrm{d}}\left[\boldsymbol{u}^{(n)}\right]\right)^{1 / 2}\right\} \\
& \leq \sqrt{2}\left(I[u(\cdot, 0)]+\frac{7}{4} L \mathcal{L}^{2} \Delta x^{2}\right)^{1 / 2} .
\end{aligned}
$$

From these evaluations for (104), we obtain

$$
\left\|\boldsymbol{e}^{(n+1)}\right\|_{\mathrm{d}-(1,2)}^{2} \leq\|\boldsymbol{e}\|_{\mathrm{d}-(1,2)}^{2}+\Delta t \lambda\left\|\tilde{\boldsymbol{e}}^{(n)}\right\|_{\mathrm{d}-(1,2)}^{2}+L\left(E_{0}\right)^{2} \Delta t
$$

Thus,

$$
\left(1-\frac{\lambda}{2} \Delta t\right)\left\|\boldsymbol{e}^{(n+1)}\right\|_{\mathrm{d}-(1,2)}^{2} \leq\left(1+\frac{\lambda}{2} \Delta t\right)\|\boldsymbol{e}\|_{\mathrm{d}-(1,2)}^{2}+L\left(E_{0}\right)^{2} \Delta t .
$$

When $\Delta t$ satisfies the condition (77), the above inequality implies

$$
\begin{aligned}
\|\boldsymbol{e}\|_{\mathrm{d}-(1,2)}^{2} & \leq \gamma\left\|\boldsymbol{e}^{(n-1)}\right\|_{\mathrm{d}-(1,2)}^{2}+\left(\frac{L\left(E_{0}\right)^{2} \Delta t}{1-\frac{\lambda}{2} \Delta t}\right) \\
& \leq \gamma^{n}\left\|\boldsymbol{e}^{(0)}\right\|_{\mathrm{d}-(1,2)}^{2}+\left(\sum_{s=0}^{n-1} \gamma^{s}\right)\left(\frac{L\left(E_{0}\right)^{2} \Delta t}{1-\frac{\lambda}{2} \Delta t}\right) \\
& \leq n \gamma^{n}\left(\frac{L\left(E_{0}\right)^{2} \Delta t}{1-\frac{\lambda}{2} \Delta t}\right)=\left(\frac{2 L\left(E_{0}\right)^{2}}{2-\lambda \Delta t}\right) T \gamma^{n} \\
& \leq \frac{3 L\left(E_{0}\right)^{2}}{2} T e^{\frac{3}{2} \lambda T},
\end{aligned}
$$

where

$$
\gamma \stackrel{\text { def }}{=} \frac{1+\frac{\lambda}{2} \Delta t}{1-\frac{\lambda}{2} \Delta t}<1+\frac{3}{2} \lambda \Delta t<e^{\frac{3}{2} \lambda \Delta t} .
$$

In the above evaluation, we have assumed that there exists no error in the initial state. From (113) and the discrete Sobolev lemma inequality (46), we obtain the theorem inequality (78). 


\section{Computation examples}

The purpose of this section is to demonstrate through some numerical computations that the proposed schemes give reasonable solutions.

\subsection{One solitary wave}

It is known that the RLW equation has a one-solitary-wave solution,

$$
u(x, t)=3 \operatorname{sech}^{2}\left(\frac{x-x_{0}-2 t}{2 \sqrt{2}}\right),
$$

where $x_{0}+2 t$ is the location of solitary wave peak. In this subsection, we set $x_{0}$ equal to 20 and an initial state for the numerical computation is given by

$$
u_{0}(x)=3 \operatorname{sech}^{2}\left(\frac{x-x_{0}}{2 \sqrt{2}}\right) .
$$

For our linear schemes, the LM and the LE schemes, we need one more initial state, $\boldsymbol{U}^{(1)}$. We use the NM scheme to compute $\boldsymbol{U}^{(1)}$ for the LM scheme and the NE scheme to compute $\boldsymbol{U}^{(1)}$ for the LE scheme.

The relative errors in mass, energy, momentum, and the peak value of the numerical solutions and computation time obtained using the four proposed schemes and the Runge-Kutta scheme at $t=40$ are listed in Table 1 . We note that the peak value of the exact solution (115) is constant and equal to 3 . The computation parameters are $\Delta x=1 / 4, \Delta t=1 / 16$, and $L=100$. The Runge-Kutta scheme is derived from ordinary differential equations of $\boldsymbol{U}: \mathbf{R} \rightarrow \mathbf{R}^{N}$,

$$
\frac{d}{d t} \boldsymbol{U}(t)=-\left(I-D_{2}\right)^{-1} D_{1}\left(\boldsymbol{U}(t)+\frac{1}{2} \boldsymbol{V}(t)\right)
$$

where $(\boldsymbol{V}(t))_{k} \stackrel{\text { def }}{=}\left(U_{k}(t)\right)^{2}$. In order to obtain new time-step solutions using our nonlinear schemes (the NM and the NE schemes), we use Newton's method.

Table 1. Relative errors in mass, energy, momentum, and peak value of numerical solutions and computation time obtained using the proposed schemes and Runge-Kutta scheme at $t=40$. Computation parameters are $\Delta x=1 / 4, \Delta t=$ $1 / 16$, and $L=100$.

\begin{tabular}{|l|cccc|c|}
\hline & mass err. & energy err. & $\begin{array}{l}\text { momentum } \\
\text { err. }\end{array}$ & $\begin{array}{l}\text { peak value } \\
\text { err. }\end{array}$ & CPU time \\
\hline NM scheme & $4.18691 \mathrm{E}-16$ & $4.40752 \mathrm{E}-03$ & $1.91544 \mathrm{E}-10$ & $1.54169 \mathrm{E}-02$ & $41 \mathrm{~m} 2.236 \mathrm{~s}$ \\
NE scheme & $2.09345 \mathrm{E}-16$ & $3.16232 \mathrm{E}-11$ & $3.97717 \mathrm{E}-06$ & $1.39999 \mathrm{E}-03$ & $30 \mathrm{~m} 8.084 \mathrm{~s}$ \\
$\begin{array}{l}\text { LM scheme } \\
\text { LE scheme }\end{array}$ & $9.21120 \mathrm{E}-15$ & $3.94501 \mathrm{E}-03$ & $1.11896 \mathrm{E}-10$ & $1.51433 \mathrm{E}-02$ & $12 \mathrm{~m} 21.904 \mathrm{~s}$ \\
$\begin{array}{l}\text { Runge-Kutta } \\
\text { scheme }\end{array}$ & $1.25607 \mathrm{E}-15$ & $3.66348 \mathrm{E}-10$ & $9.29801 \mathrm{E}-07$ & $3.44311 \mathrm{E}-05$ & $15 \mathrm{~m} 27.625 \mathrm{~s}$ \\
\hline
\end{tabular}


Energy fluctuations obtained by using our proposed schemes and the RungeKutta scheme are shown in Fig. 1. From Fig. 1, we find that the discrete energy values are conserved well, even when the non conservative schemes, the NM and the LM schemes, are used. On the other hand, the discrete energy value of the solutions obtained by using the Runge-Kutta scheme decreases monotonically.
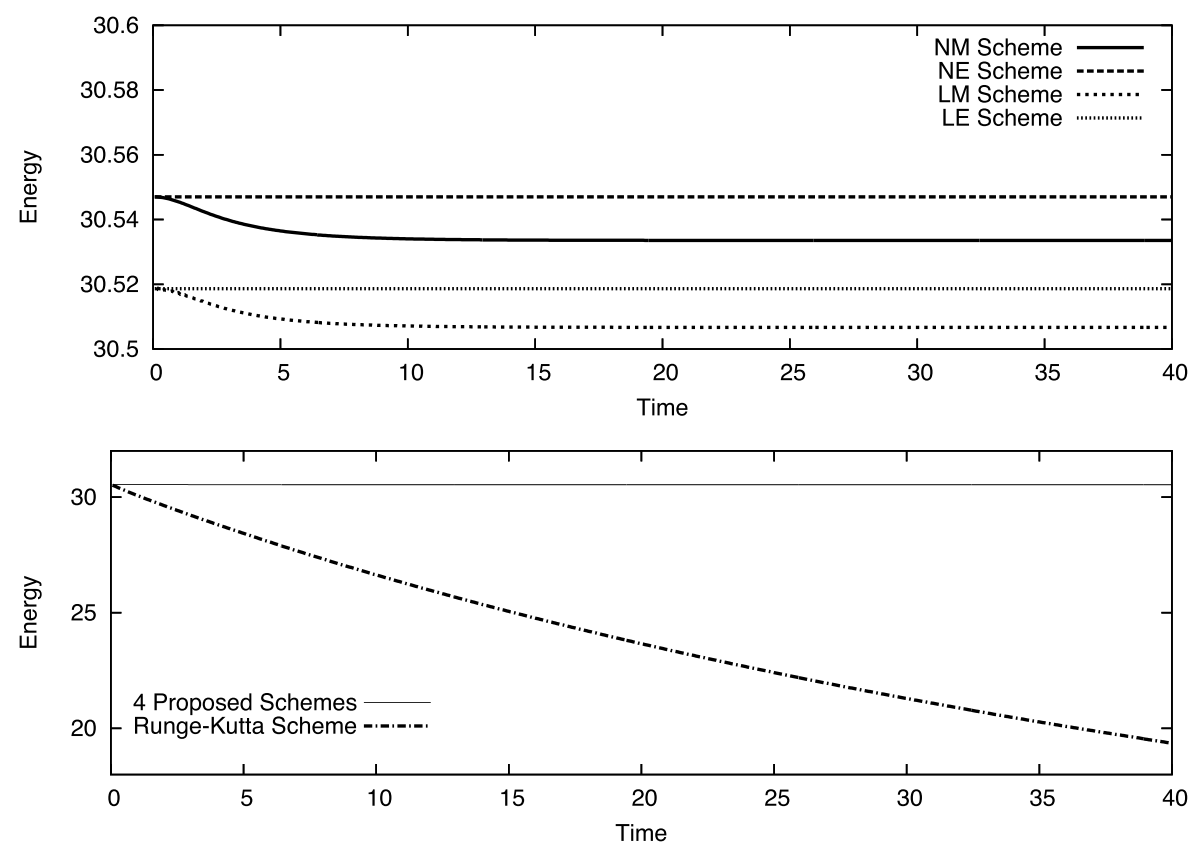

Fig. 1. Energy fluctuations obtained using our proposed schemes and Runge-Kutta scheme.

Momentum fluctuations obtained by using our proposed schemes are shown in Fig. 2. From Fig. 2, we find that the discrete momentum values are conserved well, even when the non conservative schemes, the NE and the LE schemes, are used. On the other hand, discrete momentum value of the solutions obtained by using the Runge-Kutta scheme decreases monotonically. In Fig. 2, around $t \leq 4$, there exists some vibrational fluctuation in the discrete momentum obtained by using the LE scheme. We guess that the second numerical initial state $\boldsymbol{U}^{(1)}$ is slightly inappropriate for the LE scheme.

Peak value fluctuations are indicated in Fig. 3. Thus far, no scheme, except our proposed schemes, conserves the peak value well.

Using the initial state (116), we are able to estimate the exact error values because the exact solution (115) is known. In Figs. 4 and 5, the numerical solution errors obtained by using the four proposed schemes are indicated. The left-hand side figure in Fig. 4 shows errors for the fixed time mesh size $\Delta t=1 / 16$. The $\Delta x$ for the figure are $1,1 / 2,1 / 4,1 / 8$, and $1 / 16$. The right-hand side figure in Fig. 4 

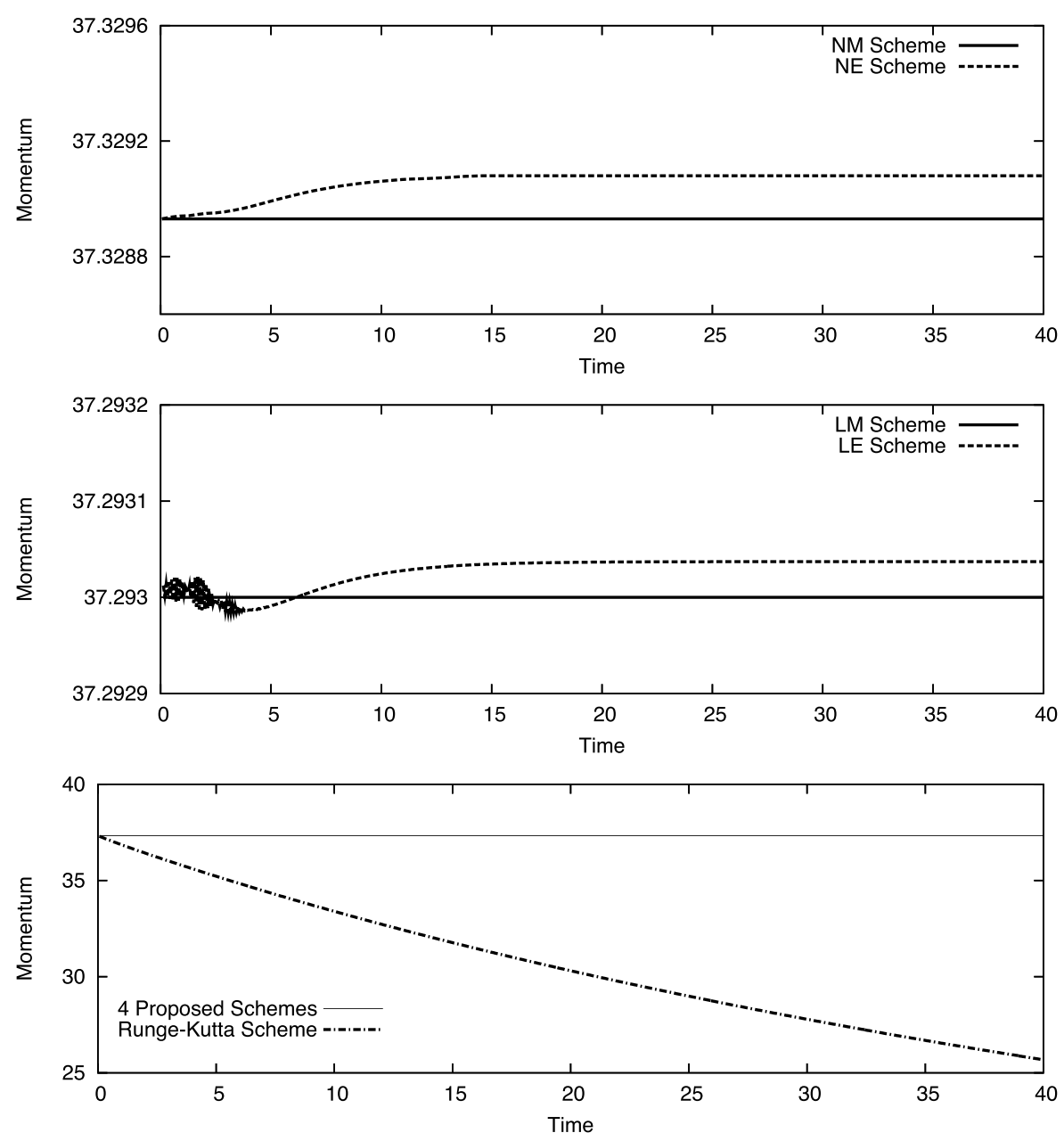

Fig. 2. Momentum fluctuations obtained using our proposed schemes and Runge-Kutta scheme.

shows the errors for fixed space mesh size $\Delta x=1 / 16$. The $\Delta t$ for the figure are $1,1 / 2,1 / 4,1 / 8$, and $1 / 16$. Fig. 5 shows the errors for $\Delta x=\Delta t$. Using the NM scheme, the max norm of errors is estimated at $O\left(\Delta x^{2}+\Delta t^{2}\right)$ using Theorem 5 , and these figures illustrate the theorem. With the other three schemes, these figures also indicate a possibility of similar error estimates. 

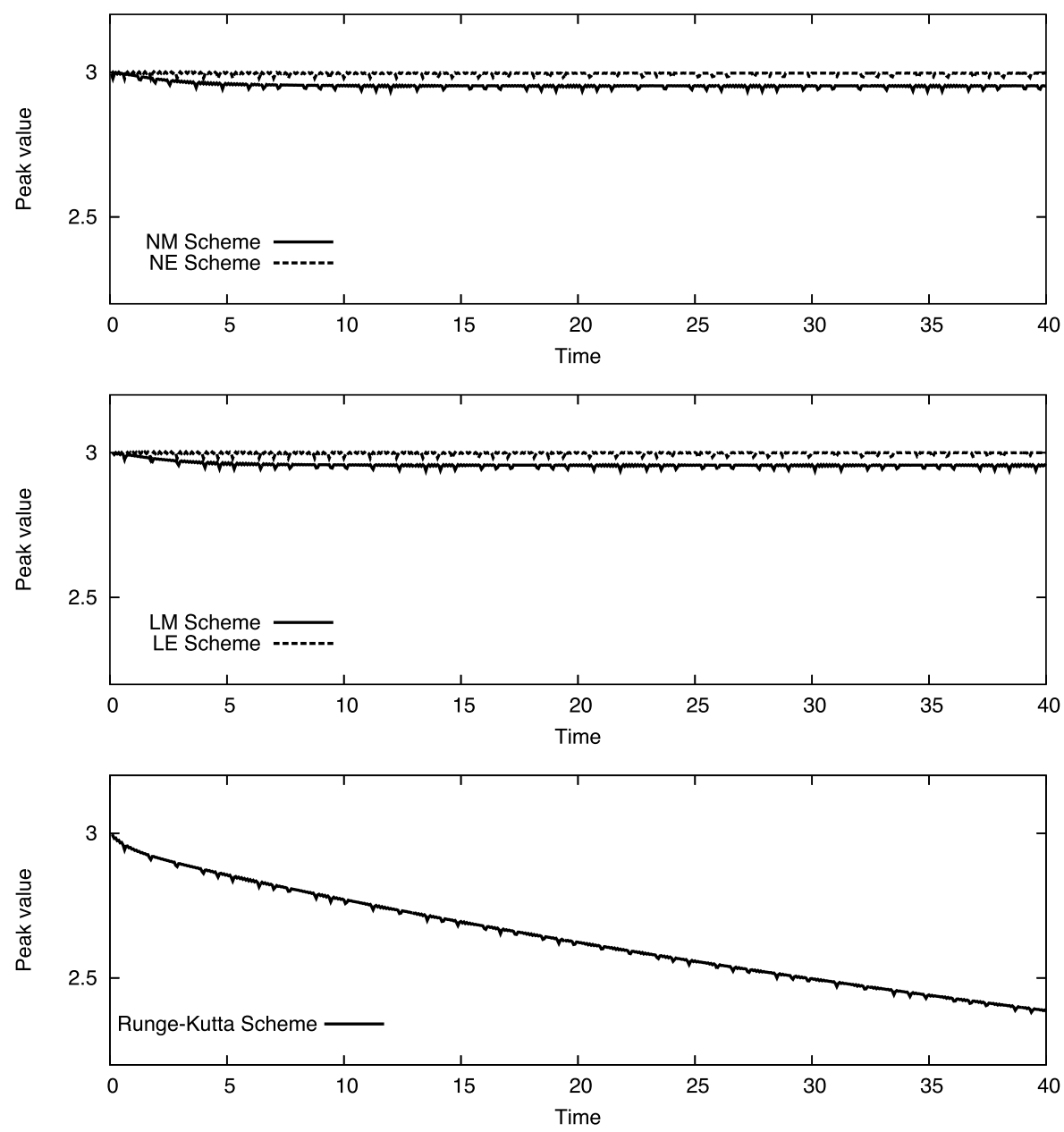

Fig. 3. Peak value fluctuations obtained using our proposed schemes and Runge-Kutta scheme.
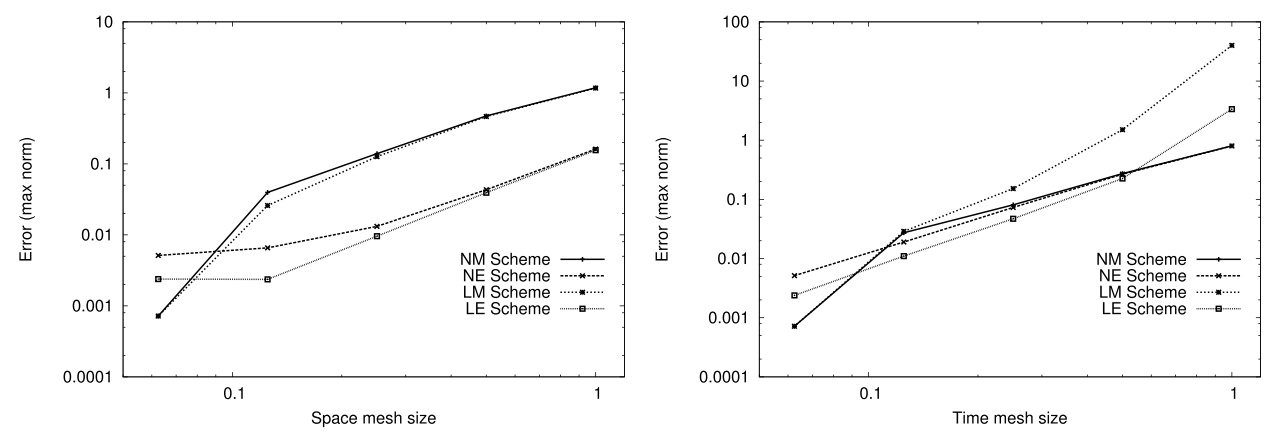

Fig. 4. Numerical solution errors (max norm) at $t=5$ using our proposed schemes. $L=$ 100 and the initial state is given by (116). Left: errors for fixed time mesh size $\Delta t=1 / 16$ and right: errors for fixed space mesh size $\Delta x=1 / 16$. 


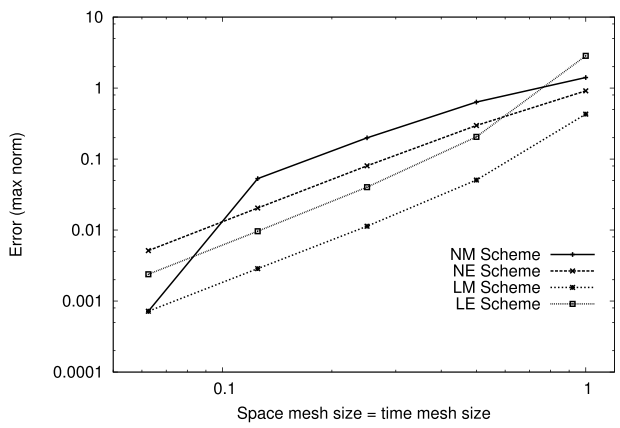

Fig. 5. Numerical solution errors (max norm) at $t=5$ for $\Delta x=\Delta t . L=100$ and the initial state is given by (116).

\subsection{Two-solitary wave}

In order to demonstrate the efficiency of our proposed schemes, we apply them to another problem with a different initial state, which is given by

$$
u_{0}(x)=3 \operatorname{sech}^{2}\left(\frac{x-x_{1}}{\sqrt{2}}\right)+\frac{3}{2} \operatorname{sech}^{2}\left(\frac{x-x_{2}}{\sqrt{3}}\right),
$$

where $x_{1}=20$ and $x_{2}=50$. This function approximates closely to a two-solitary wave with the peak locations $x_{1}$ and $x_{2}$. Fig. 6 shows the profiles of the numerical solutions, energy evolution, and momentum evolution obtained using the linear schemes for $\Delta x=1 / 5, \Delta t=1 / 32$, and $L=200$. From these figures, we find that a
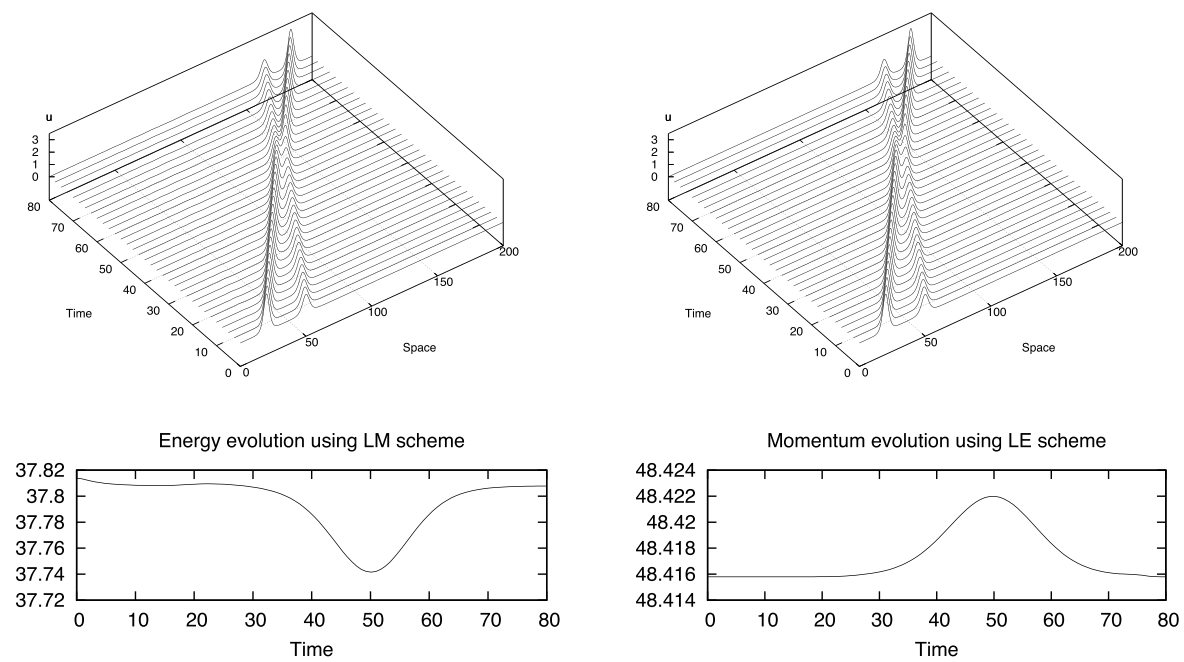

Fig. 6. Computation for two-solitary-wave initial state (118) with $\Delta x=1 / 5, \Delta t=1 / 32$, and $L=200$. Top left: profiles of numerical solutions obtained using the LM scheme, bottom left: energy evolution obtained using the LM scheme, top right: profiles of numerical solutions obtained using the LE scheme, bottom right: energy evolution obtained using the LE scheme. 
phenomenon similar to 'phase shift' occurs when two peaks of a wave collide. The non conserved quantities, i.e. energy for the LM scheme and momentum for the LE scheme, vary to some extent when two peaks of a wave collide, after which they attain their initial values.

\subsection{Undular bore}

In this subsection, we consider the parametric RLW equation

$$
\frac{\partial u}{\partial t}+\frac{\partial u}{\partial x}+u \frac{\partial u}{\partial x}-\sigma \frac{\partial^{3} u}{\partial t \partial x^{2}}=0
$$

with the initial state

$$
u(x, 0)=\frac{1}{20}\left\{1-\tanh \left(\frac{\left|x-x_{0}\right|-w}{d}\right)\right\},
$$

where $\sigma=1 / 6, x_{0}=80$, and $w=60$. The computation of this problem is a simulation of the undular bore behaviour. Here, $w$ is the bore support width and $1 / d$ is the bore slope sharpness. Some computational studies have been carried out on this equation under similar initial conditions, for example [3] and [17]. Figs. 7-9 show the profiles of the numerical solutions obtained by using the LE scheme, which is modified for the parametric RLW equation. The computation parameters are $\Delta x=1 / 5, \Delta t=1 / 16$, and $L=300$. The parameter $d$ is equal to 1,2 , and 3 in
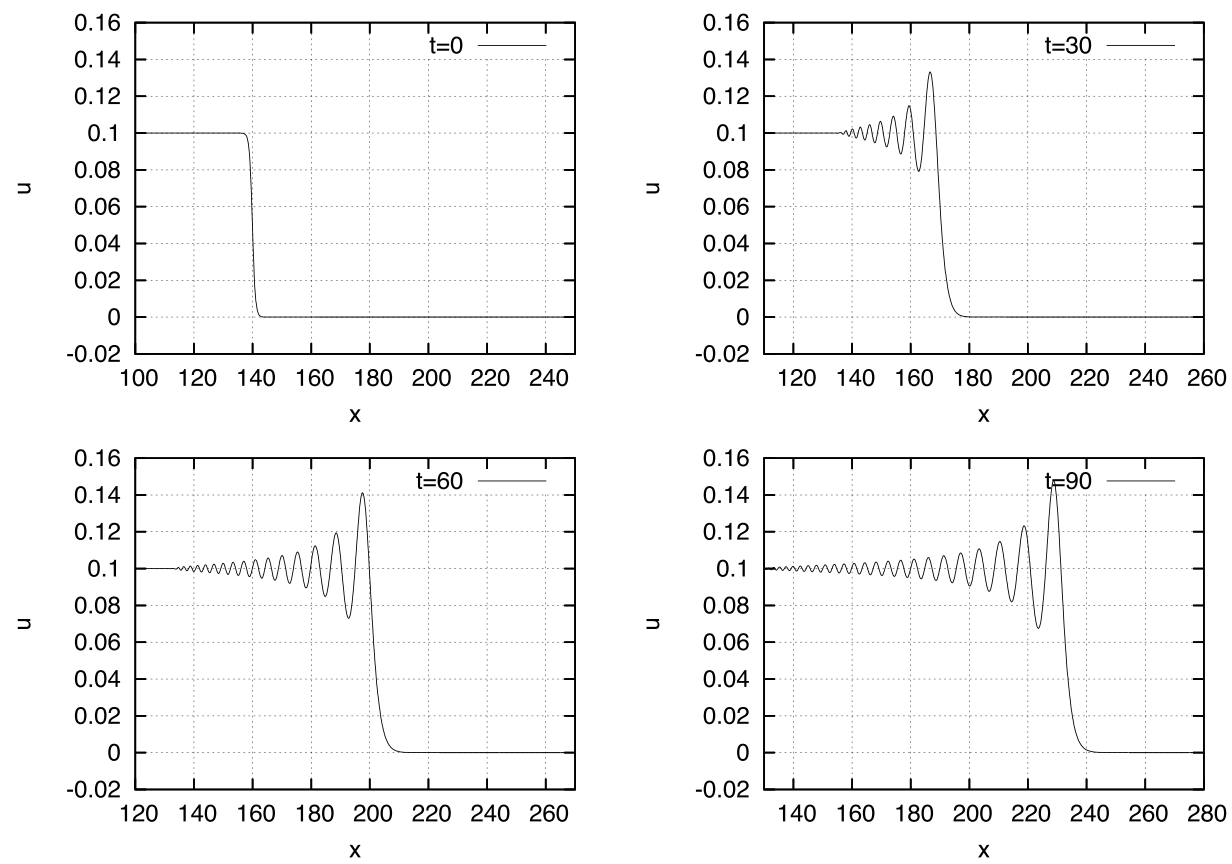

Fig. 7. Numerical solutions for undular bore problem (119) for (120) and $d=1$. Computation parameters are $\Delta x=1 / 5, \Delta t=1 / 16$, and $L=300$. 

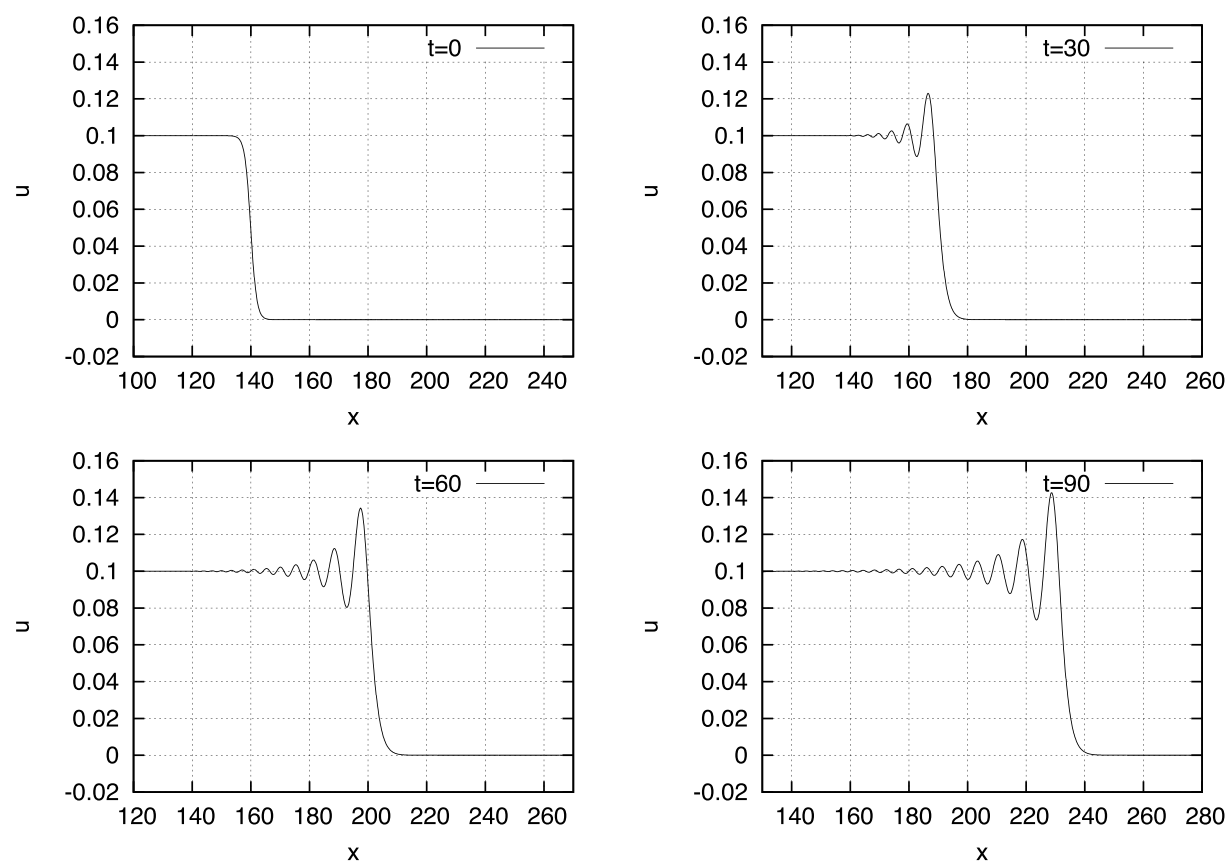

Fig. 8. Numerical solutions for undular bore problem (119) for (120) and $d=2$.

Computation parameters are $\Delta x=1 / 5, \Delta t=1 / 16$, and $L=300$.
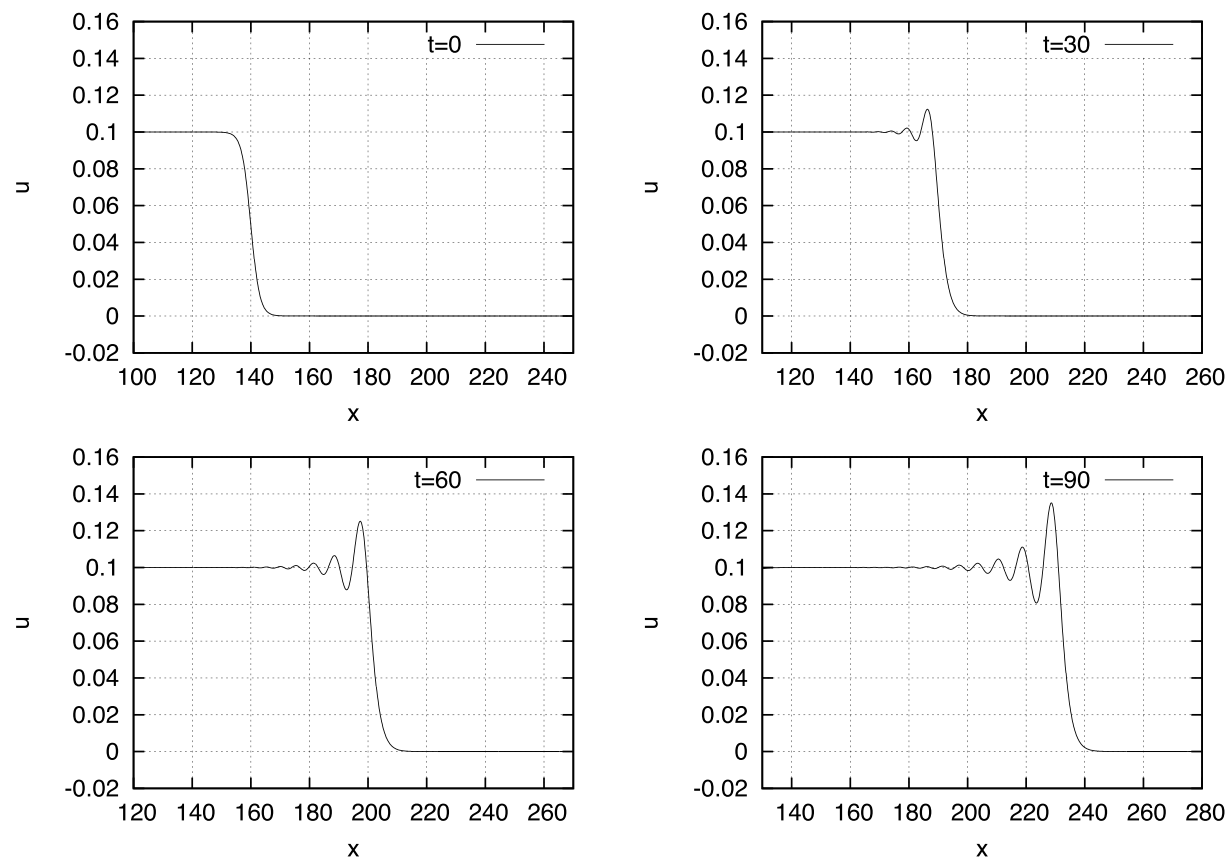

Fig. 9. Numerical solutions for undular bore problem (119) for (120) and $d=3$. Computation parameters are $\Delta x=1 / 5, \Delta t=1 / 16$, and $L=300$. 
Figs. 7, 8, and 9 respectively. From these figures, we can say that undulation becomes gentler as $d$ increases. These computation results agree with those obtained by Dağ [3]. This agreement proves that the computations carried out by using our schemes are reliable.

\section{Conclusion}

We have proposed four new finite difference schemes for the RLW equation, which conserve either discrete energy or discrete momentum. Using the nonlinear scheme that conserves momentum and mass, we show that it has unique solutions under some condition, the solutions are stable in the maximum norm, and the errors in the solutions are essentially estimated at $O\left(\Delta x^{2}+\Delta t^{2}\right)$. Through some numerical computations, the efficiency and robustness of our proposed schemes have been demonstrated.

\section{Appendix. Discrete calculus under discrete boundary condition}

Some relationships between difference operators and summations are described in this appendix. First, we describe the summations of difference equal to zero under the discrete boundary condition (7). The summations are given as

$$
\begin{aligned}
& \sum_{k=0}^{N-1} \delta_{k}^{\langle 1\rangle} f_{k} \Delta x=\frac{1}{2}\left\{\left(f_{N}-f_{0}\right)+\left(f_{N-1}-f_{-1}\right)\right\}=0 \\
& \sum_{k=0}^{N-1} \delta_{k}^{\langle 2\rangle} f_{k} \Delta x=\frac{1}{\Delta x}\left\{\left(f_{N}-f_{0}\right)-\left(f_{N-1}-f_{-1}\right)\right\}=0
\end{aligned}
$$

for $\boldsymbol{f} \in \mathbf{R}^{(N)}$. The following relationship is 'summation by parts,' which corresponds to integration by parts:

$$
\sum_{k=0}^{N-1} f_{k}\left(\delta_{k}^{+} g_{k}\right) \Delta x+\sum_{k=0}^{N-1}\left(\delta_{k}^{-} f_{k}\right) g_{k} \Delta x=f_{N-1} g_{N}-f_{-1} g_{0}=0
$$

for $\boldsymbol{f}, \boldsymbol{g} \in \mathbf{R}^{(N)}$. The application of summation by parts yields

$$
\begin{aligned}
& \sum_{k=0}^{N-1} f_{k}\left(\delta_{k}^{\langle 1\rangle} g_{k}\right) \Delta x+\sum_{k=0}^{N-1}\left(\delta_{k}^{\langle 1\rangle} f_{k}\right) g_{k} \Delta x=0 \\
& \sum_{k=0}^{N-1} f_{k}\left(\delta_{k}^{\langle 1\rangle} f_{k}\right) \Delta x=0
\end{aligned}
$$

for $\boldsymbol{f}, \boldsymbol{g} \in \mathbf{R}^{(N)}$. 
Acknowledgement. We thank M. Mori, M. Sugihara, and K. Murota for their helpful advice and discussions.

\section{References}

[ 1 ] T.B. Benjamin, J.L. Bona and J.J. Mahony, Model equations for long waves in nonlinear dispersive systems. Philos. Trans. R. Soc. London Ser. A, 272 (1972), 47-78.

[2] İ. Dağ and M.N. Özer, Approximation of the RLW equation by the least square cubic B-spline finite element method. Appl. Math. Model., 25 (2001), 221-231.

[ 3 ] İ. Dağ, B. Saka and D. Irk, Application of cubic B-splines for numerical solution of the RLW equation. Appl. Math. Comput., 159 (2004), 373-389.

[ 4 ] A. Durán and M.A. López-Marcos, Conservative numerical methods for solitary wave interactions. J. Phys. A: Math. Gen., 36 (2003), 7761-7770.

[5] J.C. Eilbeck and G.R. McGuire, Numerical study of the regularized long-wave equation, I: numerical methods. J. Comput. Phys., 19 (1975), 43-57.

[6] J.C. Eilbeck and G.R. McGuire, Numerical study of the regularized long-wave equation, II: interaction of solitary waves. J. Comput. Phys., 23 (1977), 63-73.

[ 7 ] D. Furihata, Finite difference schemes for $\frac{\partial u}{\partial t}=\left(\frac{\partial}{\partial x}\right)^{\alpha} \frac{\delta G}{\delta u}$ that inherit energy conservation or dissipation property. J. Comput. Phys., 156 (1999), 181-205.

[ 8 ] D. Furihata, Finite difference schemes for nonlinear wave equation that inherit energy conservation property. J. Comput. Appl. Math., 134 (2001), 37-57.

[ 9 ] D. Furihata and T. Matsuo, A stable, convergent, conservative and linear finite difference scheme for the Cahn-Hilliard equation. Japan J. Indust. Appl. Math., 20 (2003), 65-85.

[10] D. Furihata and M. Mori, A stable finite difference scheme for the Cahn-Hilliard equation based on the Lyapunov functional. ZAMM Z. angew. Math. Mech., 76 (1996), 405-406.

[11] T. Hanada, N. Ishimura and M. Nakamura, Stable finite difference scheme for a model equation of phase separation. Appl. Math. Comput., 151 (2004), 95-104.

[12] T. Ide, C. Hirota and M. Okada, Generalized energy integral for $\partial u / \partial t=\delta G / \delta u$ and its finite difference schemes by means of discrete variational method and an application to Fujita problem. Adv. Math. Sci. Appl., 12 (2002), 755-778.

[13] F. John, Lectures on Advanced Numerical Analysis. Gordon and Breach, New York, 1967.

[14] T. Matsuo and D. Furihata, Dissipative or conservative finite difference schemes for complexvalued nonlinear partial differential equations. J. Comput. Phys., 171 (2001), 425-447.

[15] T. Matsuo, M. Sugihara and M. Mori, A derivation of a finite difference scheme for the nonlinear Schrödinger equation by the discrete variational derivative (in Japanese). Trans. Japan Soc. Indust. Appl. Math., 8 (1998), 405-426.

[16] P.J. Olver, Euler operators and conservation laws of the BBM equation. Math. Proc. Camb. Phil. Soc., 85 (1979), 143-160.

[17] D.H. Peregrine, Calculations of the development of an undular bore. J. Fluid Mech., 25 (1966), 321-330. 